\title{
Self-Excited Supersonic Cavity Flow \\ Instabilities as Aerodynamic Noise Sources
}

\author{
A. Rona $*, 1$ \\ Department of Engineering, University of Leicester, UK
}

\begin{abstract}
A turbulent cavity flow at Mach 1.5 and 2.5 is modelled to study the flow instability and the associated aerodynamic noise generation. The short-time averaged NavierStokes equations, coupled with a $k-\omega$ turbulence model, are solved to predict the large-scale time-dependent flow. Values of the cavity wall pressure, drag, streamwise velocity and density are in good in agreement with past measurements and the results of other computations. The noise generation physics of the unsteady flow is addressed by estimating the noise source strength in a Lighthill acoustic analogy. The time-dependent flow predictions highlighted the upstream and downstream cavity edges as areas of large flow unsteadiness. The same areas are identified by the acoustic analogy as the dominant noise source regions in this flow.
\end{abstract}

Key words: Unsteady aerodynamics, cavity flow, noise sources

PACS: 43.28.+h, 47.11.-j, 47.27.Sd, 47.40.Hg

\footnotetext{
* Corresponding Author. Address: Department of Engineering, University of Leicester, Leicester LE1 7RH, Tel. +44-116-252 2510, Fax: +44-116-252 2525.

Email address: ar45@le.ac.uk (A. Rona ).

URL: http://www.le.ac.uk/eg/ar45 (A. Rona ).

1 Lecturer.
}

Preprint submitted to the international journal of aeroacoustics 17 October 2006 


\section{Introduction}

A numerical investigation is performed on the time-mean and time-dependent characteristics of the compressible turbulent flow over a rectangular cavity or enclosure. At certain flow regimes [1], the flow-geometry interaction occurring in a cavity onsets large-scale self-excited instabilities that dominate the aerodynamic flow field. In this field, the time-dependent pressure is oscillatory along the solid boundaries and generates time-dependent drag. Strong tonal noise is also generated by the aerodynamic instabilities. The physics of noise production is based on Lighthill's acoustic analogy. This study addresses by numerical investigation the noise source characteristics based on the timeaveraged Lighthill stress tensor distribution, derived from the time-dependent aerodynamic results. This approach attempts to capture through the acoustic analogy the aerodynamic noise sources responsible for the sound perceived in the acoustic far-field. This investigation complements the available literature on the aerodynamics and near-field acoustics of self-sustained open cavity flows [2-5], giving some further information on the source strength of the noise perceived at more than 20 cavity lengths away from the enclosure.

Zhang [6,7] discussed the flow unsteadiness developing over a cavity or enclosure. A turbulent boundary layer approaches the enclosure towards the upstream edge (Fig. 1). At this location, the geometry induced flow separation forms a shear layer. The latter is unstable, developing large-scale self-sustained instabilities that characterize the unsteady aerodynamic field. Aerodynamic pressure fluctuations inside the enclosure accompany the observed shear layer unsteadiness and provide the feed-back for the onset of a stable self-sustained oscillatory flow regime. The flow instability is responsible for wall pressure 
fluctuations, pressure drag and aerodynamic noise radiation.

In this study, a rectangular enclosure of length to depth ratio 3 is tested at free stream Mach numbers $\left(M_{\infty}\right)$ of 1.5 and 2.5. Measurements and past numerical predictions by Zhang [6,7] are available for comparison. Charwat et al. [8] identified a 'closed' flow regime where the shear layer reattaches to the central section of the cavity floor, and an 'open' flow geometry where the reattachment point is the downstream edge. The flow geometry considered here is 'open' and approximately two-dimensional (2-D).

Even the simple geometry of a rectangular cavity develops a complex flow pattern that is the object of on-going fundamental research (e.g. Jeng \& Payne [9] and Zhang et al. [10]). Practical applications of related cavity flows include slotted wall wind tunnels, slotted flumes, bellows-type pipe geometries, gate slots, spacecraft, and aircraft components. The subject is reviewed by Rockwell \& Naudascher [2], Blake \& Powell [3], Colonius [4], Grace [11] and by Rowley \& Williams [12].

In the time-averaged flow, the presence of the shear layer and its rate of growth, the pressure distribution around the walls, the characteristics of the in-coming boundary layer and of the re-attached flow downstream of the cavity all influence the cavity mean drag. A quantitative prediction is attempted of the salient time-averaged features to estimate the cavity mean drag.

In the time-dependent flow, a qualitative analysis is presented on the convective amplification of the large-scale instability in the shear layer and of the accompanying unsteady convected vorticity. The flow-geometry interaction at the downstream cavity edge dominates the pressure fluctuations throughout the cavity. Past studies have used the knowledge of such interaction to reduce 
the unsteady aerodynamic loads of a model enclosure $[13,14]$. This study aims to relate such flow physics to the sources of the aerodynamic noise perceived in the acoustic far-field.

Aerodynamic noise is generated as a by-product of the flow instability. Cavity noise measurements by Block [15] and numerical studies by Hardin \& Pope [16] highlighted the tonal characteristics of the radiated sound field at certain flow regimes. For the selected test cases the noise characteristic wavelength $(\lambda)$ is greater than the cavity characteristic dimension $(D)$. Flow-acoustic interaction inside the cavity is limited by the size of the enclosure $(\lambda<D)$ and hence the unsteady flow can be regarded as a compact noise source. In the acoustic far-field, at a distance of 20 or more cavity lengths away from the enclosure, the pressure fluctuation perceived by an observer as aerodynamic noise is the integral effect of the Lighthill stress tensor field. This can be extracted from the time-dependent aerodynamic predictions.

The present study focuses on the large-scale turbulent structure in the cavity responsible for aerodynamic noise generation. Lighthill's acoustic analogy is followed to identify the acoustically active regions in the flow field. The physics of noise generation is addressed as a by-product of the time-dependent flow. These results contribute to the understanding of cavity noise generation, which is the basis for developing successful noise control strategies.

\section{Flow Conditions}

The geometry and flow conditions describe a longitudinal rectangular cavity driven by a turbulent shear layer, studied in Zhang [6,7]. The depth of the 
cavity $D$ is $15 \mathrm{~mm}$ and the length of the cavity $L$ is $45 \mathrm{~mm}$. The geometry is tested at free stream Mach numbers $\left(M_{\infty}\right)$ of 1.5 and 2.5. At $M_{\infty}=1.5$ the free stream temperature $\left(T_{\infty}\right)$, pressure $\left(p_{\infty}\right)$, density $\left(\rho_{\infty}\right)$, stagnation temperature $\left(T_{s}\right)$ and stagnation pressure $\left(p_{s}\right)$ are $200 \mathrm{~K}, 53.801 \mathrm{kN} / \mathrm{m}^{2}, 0.9373 \mathrm{~kg} / \mathrm{m}^{3}$, $288.5 \mathrm{~K}$, and $197.51 \mathrm{kN} / \mathrm{m}^{2}$ respectively. At $M_{\infty}=2.5$ the corresponding values are $128 \mathrm{~K}, 17.390 \mathrm{kN} / \mathrm{m}^{2}, 0.4701 \mathrm{~kg} / \mathrm{m}^{3}, 288.5 \mathrm{~K}$, and $297.12 \mathrm{kN} / \mathrm{m}^{2}$. A turbulent boundary layer approaches the enclosure upstream edge. The boundary layer thickness $(\delta)$ and momentum thickness $\left(\delta_{2}\right)$ are respectively $5 \mathrm{~mm}, 0.417 \mathrm{~mm}$ at $M_{\infty}=1.5$, and $5 \mathrm{~mm}$ and $1.290 \mathrm{~mm}$ at $M_{\infty}=2.5$. The flow Reynolds number ( $R e$ ) based on the cavity depth is $4.5 \times 10^{5}$ in both cases.

Non-dimensional short-time averaged variables () are used in the numerical model. The density $(\rho)$, velocity vector $(\boldsymbol{u})$, pressure $(p)$, specific enthalpy $(h)$, turbulent kinetic energy $(k)$, temperature $(T)$, and dynamic viscosity $(\mu)$ are normalized by the free stream conditions $\left(\rho_{\infty}, U_{\infty}, T_{\infty}, \mu_{\infty}\right)$; the specific turbulent dissipation rate $(\omega)$ by $U_{\infty} R e / D$, and time by $D / U_{\infty}$.

\section{$3 \quad$ Numerical Model}

\subsection{Aerodynamic model}

A combined deterministic and stochastic approach is followed to model the cavity aerodynamic flow. The flow visualization by Zhang [6] in Fig. 9(a) indicates that the aerodynamic field can be regarded as large-scale time-evolving structures in a background of random turbulence. These large-scale flow instabilities are obtained from the selective amplification of flow disturbances in the cavity. The excited modes are the eigenmodes of the cavity flow, as 
shown by the analysis of Bilanin \& Covert [17]. The enclosure geometry and the inlet boundary layer profile are the boundary conditions that determine the eigenmodes and, more generally, the frequency and wavenumber response characteristics of the cavity. The observed large-scale structures develop in the shear layer spanning the cavity open surface and are Kelvin-Helmoltz type instabilities. They are therefore problem-specific and their initial growth is essentially a deterministic inviscid process. The large-scale instability growth can be modelled by inviscid instability analysis methods as in Tam \& Block [18], or by a time-dependent numerical solution of the Euler equations as in Zhang \& Edwards [19] and Rona \& Dieudonné [20].

Measurements by Zhang [6] show that the large-scale instabilities are generated at the cavity upstream edge. These are convectively amplified, impinging against the downstream edge within approximately one fundamental instability mode wavelength $(\lambda)$ from their point of origin. During convection over the cavity open surface, the small flow perturbations grow to a finite amplitude and the modes saturate, displaying convective mode non-linearities. The large-scale instabilities reach the downstream edge having covered just one fundamental mode wavelength, which is unlikely to be sufficient to allow the dominant instability modes to decay in smaller eddies and establish a fully developed turbulent flow. The large scale structure can still be readily identified on the trailing edge plate in Zhang [6], at a streamise distance greater than $6 D$ from the upstream edge. The accompanying wall pressure measurements in Zhang [6] confirm a power spectral density dominated by low frequency isolated tones over a lower amplitude broad-band turbulent contribution, decaying with frequency. Further downstream, away from the enclosure, the convected instabilities are expected to decay, creating a tur- 
bulent kinetic energy cascade to the higher wavenumbers and the turbulent kinetic energy is eventually dissipated by viscous stresses in the dissipation sub-range, at the Kolmogorov length scale. As the flow is supersonic, this downstream flow regime does not significantly affect the upstream cavity.

A separation of kinetic energy length scales approach is adopted to obtain a numerical model of the cavity flow. The model exploits the modest energy transfer (interaction) between the large-scale structure and the background of fine-scale turbulence in the selected cavity test cases. A time-dependent prediction of the large-scale structure is performed using an explicit timemarching finite-volume method. In the numerical model, the grid size does not allow to resolve directly the small-scale structure in the dissipation sub-range and the Wilcox [21] $k-\omega$ two-equation model is used to provide turbulence closure.

The large-scale structure fluctuations can be extracted from the flow history by averaging over a time that is short compared to the characteristic period of the large eddies in the flow field and long compared to the fluctuations of the small-scale turbulence. The instantaneous value of any flow variable $f$ is thus split in a short-time averaged component $\tilde{f}$ and a fluctuation due to small-scale turbulence $f^{\prime \prime}$, which is treated as a stochastic variable. This process is called short-time averaging. For simplicity, terms like $\widetilde{\rho}^{\prime \prime} T^{\prime \prime}$ are not modelled, since, for instance, heat transfer effects mainly depend on the timemean temperature gradient.

For steady flow problems, such as the compressible turbulent boundary layer flow of Mabey \& Sawyer [22], the short-time averaged method is identical to an explicit Reynolds averaged method and converges to a steady state. 
In such fully developed turbulent flow, all kinetic energy length scales are modelled by the $k$ and $\omega$ equations. In the cavity flow, a continuous turbulent spectrum is not achieved at the downstream edge and the $k-\omega$ model covers a different role than in steady flow problems. First, it models the statistics of the downstream evolution of the fine-scale turbulence, generated by the upstream boundary layer. The wavenumber range of these flow features is above the cavity eigenmodes range. Second, it augments the effects of laminar viscosity to control the saturation of the enclosure eigenmodes. In the timedependent cavity model, the short-time velocity gradient is used to estimate the turbulent stress tensor, while the mean velocity gradient would be used for the Reynolds stresses in a time-mean approach. This attempts to filter the large-scale kinetic energy contribution in the small-scale turbulence model.

Consider a compressible turbulent flow governed by the short-time averaged Navier-Stokes equations (Lilley et al. [23]) written in vector form. The turbulent stress tensor is estimated using the $k-\omega$ two-equation model of Wilcox [21]:

$$
\frac{\partial}{\partial \tau} \tilde{\boldsymbol{U}}+\nabla \cdot\left(\tilde{\boldsymbol{F}}_{i}+\tilde{\boldsymbol{F}}_{t}\right)+\tilde{\boldsymbol{S}}=0
$$

where

$$
\begin{aligned}
\tilde{\boldsymbol{U}}= & {\left[\tilde{\rho}, \tilde{\rho} \tilde{\boldsymbol{u}}, \tilde{\rho}\left(\tilde{e}_{s}+\tilde{k}\right), \tilde{\rho} \tilde{k}, \tilde{\rho} \tilde{\omega}\right]^{T}, } \\
\tilde{\boldsymbol{F}}_{i}= & {\left[\tilde{\rho} \tilde{\boldsymbol{u}}, \tilde{\rho} \tilde{\boldsymbol{u}} \tilde{\boldsymbol{u}}+\tilde{p} \boldsymbol{I}, \tilde{\rho} \tilde{\boldsymbol{u}}\left(\tilde{h}_{s}+\tilde{k}\right), \tilde{\rho} \tilde{\boldsymbol{u}} \tilde{k}, \tilde{\rho} \tilde{\boldsymbol{u}} \tilde{\omega}\right]^{T} } \\
\tilde{\boldsymbol{F}}_{t}= & {\left[0,-(\tilde{\boldsymbol{\tau}}+\tilde{\boldsymbol{t}}),-\left(\tilde{\boldsymbol{q}}+\tilde{\boldsymbol{q}}_{t}\right)-(\tilde{\boldsymbol{\tau}}+\tilde{\boldsymbol{t}}) \cdot \tilde{\boldsymbol{u}}-\left(\tilde{\mu}_{l}+\sigma^{*} \tilde{\mu}_{t}\right) \nabla \tilde{k} / R e,\right.} \\
& \left.-\left(\tilde{\mu}_{l}+\sigma^{*} \tilde{\mu}_{t}\right) \nabla \tilde{k} / R e,-\left(\tilde{\mu}_{l}+\sigma \tilde{\mu}_{t}\right) \nabla \tilde{\omega} / R e\right]^{T}, \\
\tilde{\boldsymbol{S}}= & {\left[0,0,0, \beta^{*} \tilde{\rho} \tilde{k} \tilde{\omega} R e-\tilde{\boldsymbol{t}}: \nabla \tilde{\boldsymbol{u}}, \beta^{* *} \tilde{\rho} \tilde{\omega}^{2} R e-\alpha(\tilde{\omega} / \tilde{k}) \tilde{\boldsymbol{t}}: \nabla \tilde{\boldsymbol{u}}\right]^{T} . }
\end{aligned}
$$

The relationship for a perfect gas $\tilde{p}=\tilde{\rho} \tilde{T} / \gamma M_{\infty}^{2}$ completes the governing equa- 
tions. The eddy viscosity $\left(\tilde{\mu}_{t}\right)$, dynamic viscosity $\left(\tilde{\mu}_{l}\right)$, viscous stress tensor $(\tilde{\boldsymbol{\tau}})$, turbulent stress tensor $(\tilde{\boldsymbol{t}})$, heat flux vectors $\left(\tilde{\boldsymbol{q}}, \tilde{\boldsymbol{q}}_{t}\right)$, specific stagnation energy $\left(\tilde{e}_{s}\right)$, and specific internal energy $(\tilde{e})$ are defined in the following auxiliary relations:

$$
\begin{aligned}
\tilde{\mu}_{t} & =\tilde{\rho} \tilde{k} / \tilde{\omega} \\
\tilde{\mu}_{l} & =c_{1} \frac{\tilde{T}^{3 / 2}}{\tilde{T}+c_{2}}, \\
\tilde{\boldsymbol{\tau}} & =\tilde{\mu}_{l}(\nabla \tilde{\boldsymbol{u}}+\tilde{\boldsymbol{u}} \nabla-2 / 3 \boldsymbol{I} \nabla \cdot \tilde{\boldsymbol{u}}) / R e \\
\tilde{\boldsymbol{t}} & =\tilde{\mu}_{t}(\nabla \tilde{\boldsymbol{u}}+\tilde{\boldsymbol{u}} \nabla-2 / 3 \boldsymbol{I} \nabla \cdot \tilde{\boldsymbol{u}}) / R e-2 / 3 \boldsymbol{I} \tilde{\rho} \tilde{k}, \\
\tilde{\boldsymbol{q}} & =\frac{1}{(\gamma-1) M_{\infty}^{2} \operatorname{Re} \frac{\tilde{\mu}_{l}}{\operatorname{Pr}} \nabla \tilde{T},} \\
\tilde{\boldsymbol{q}}_{t} & =\frac{1}{(\gamma-1) M_{\infty}^{2} \operatorname{Re}} \frac{\tilde{\mu}_{t}}{\operatorname{Prt}} \nabla \tilde{T}, \\
\tilde{e}_{s} & =\tilde{e}+\tilde{\boldsymbol{u}} \cdot \tilde{\boldsymbol{u}} / 2, \\
\tilde{e} & =\tilde{T} /\left[\gamma(\gamma-1) M_{\infty}^{2}\right] .
\end{aligned}
$$

The constant coefficients $\left(c_{1}, c_{2}\right)$ in the non-dimensional form of the Sutherland law are $1.458 \times 10^{-6} \sqrt{T_{\infty}} / \mu_{\infty}$ and $110.4 / T_{\infty}$ respectively. The Prandtl number $(P r)$ and the turbulent Prandtl number $(P r t)$ are 0.71 and 0.90 respectively. The turbulence closure coefficients $\left(\alpha, \beta^{*}, \beta^{* *}, \sigma, \sigma^{*}\right)$ in the $k-\omega$ model are modified for the effects of compressibility. The details can be found in Wilcox [21].

A $160 \times 200$ rectangular grid defines the control volumes for the application of the conservation laws. A finer $320 \times 400$ grid is also used to assess the grid size effect on the aerodynamic predictions. The computational domain covers a flow area of $12 D$ and $5 D$ in the streamwise and normal directions, the unit cell dimensions $\left(\Delta y_{1}, \Delta y_{2}\right)$ are $(0.075 D, 0.025 D)$ and $(0.0375 D, 0.0125 D)$ for the $160 \times 200$ and $320 \times 400$ grids respectively.

The Roe [24] flux difference split approximate Riemann method estimates the 
inviscid fluxes $\left(\tilde{\boldsymbol{F}}_{i}\right)$ at the unit volume boundaries. The second-order space accurate extension is implemented. The MinMod inviscid flux limiter function is adopted to preserve monotonicity. The inviscid flux components of the $k-\omega$ equations are included as in Rona [25], following the same procedure of Roe. Second-order central difference is used to estimate the turbulent fluxes $\left(\tilde{\boldsymbol{F}}_{t}\right)$ that are integrated with the inviscid ones in finite-volume form. This compact integration strategy upgrades the operator split approach by Zhang [7], enhancing the efficiency of the numerical quadrature in the model. A multi-step Runge-Kutta explicit time-marching method advances the flow prediction in time. Non-standard coefficients are used to enhance the scheme stability. These are $b_{1}=0.12, b_{1}=0.25, b_{3}=0.5$, and $b_{4}=1.0$. Specifically, an inviscid simulation is stable up to a Courant number $(C F L=\tilde{c} \Delta \tilde{\tau} / \Delta y)$ of 2.0 (Manna [26]), while the same scheme with two-equation turbulence closure was tested to be stable at $C F L=0.53$. The method is second-order time accurate. The flow history is advanced with a 0.01 non-dimensional fixed time step $(\Delta \tilde{\tau})$ over which the flow is short-time averaged. At $M_{\infty}=1.5$ this corresponds to $C F L=0.27$ and $C F L=0.53$ for the low and high density grids respectively. At $M_{\infty}=2.5$ the corresponding values are $0.16 C F L$ and $0.32 C F L$. The Nyquist Strouhal number $\left[S t r=f D / U_{\infty}\right]$ is 100 , which corresponds to a $2.83 \mathrm{MHz}$ Nyquist frequency at $M_{\infty}=1.5$ and $3.78 \mathrm{MHz}$ at $M_{\infty}=2.5$.

At the inflow boundary (b1) the mass flux remains constant throughout the computation and is similar to the upstream boundary condition used by Bastin [27] for the case of a supersonic uniform stream. In the unsteady cavity flow, upstream propagating disturbances are present in the boundary layer. The upstream effect is limited below the sonic line to a distance less than one boundary layer thickness. This is difficult to quantify, but since the distur- 
bances are damped while propagating from the upstream cavity edge $\left(y_{1}=0\right)$ to the inflow boundary $\left(y_{1}=-3 D\right)$, the selected boundary condition appears to be adequate (Rona [25]). Along the solid walls $(b 2-b 6)$ a no-slip condition is imposed. At the outflow (b7) all conservative variables are extrapolated assuming constant gradients in space (first order extrapolation). Non-reflecting boundary conditions (Zhang [7]) apply on (b8). Along this latter computational boundary, the flow is assumed uniform and parallel and the conservative variables are set equal to their interior values and are constant along the outgoing Mach wave.

The flow state at the beginning of the computation is described in Fig. 2. The inflow condition is imposed in the flow field above the cavity. Inside the cavity, stagnation temperature conditions apply and $\boldsymbol{u}=\mathbf{0}$. As in Zhang [7], the flow is perturbed over the cavity open end to facilitate the onset of the self-sustained unsteady flow regime. This is reached after $\tilde{\tau} \sim \mathbf{O}(50)$. The computation is then extended to $\tilde{\tau} \sim \mathbf{O}(150)$ to estimate the time-mean flow and monitor the aerodynamic pressure on the cavity walls. This corresponds to approximately 10 shear-layer oscillations at the fundamental mode. The wall pressure history is predicted at $y_{1}=0.33 D$ and $y_{1}=2.33 D$ on the floor of the enclosure, and at $y_{1}=3.66 D$ and $y_{1}=5.66 D$ above the downstream cavity edge. The sound pressure level at the enclosure walls and the cavity form drag are also estimated.

\subsection{Noise source prediction}

Lighthill's acoustic analogy is used to determine the noise generation from the predicted unsteady flow. The main contributions to the far-field acoustic 
radiation are expected from the momentum flux fluctuations in the largescale structure. The sources of noise can be found by short-time averaging the Lighthill [28] governing equation for aerodynamic noise. This equation is

$$
\square^{2} \tilde{\rho}=\nabla \cdot \nabla \cdot \tilde{\boldsymbol{T}}
$$

where the tensor $\tilde{\boldsymbol{T}}=\tilde{\rho} \tilde{\boldsymbol{u}} \tilde{\boldsymbol{u}}-(\tilde{\boldsymbol{\tau}}+\tilde{\boldsymbol{t}})+\left(\tilde{p}-c_{\infty}^{2} \tilde{\rho}\right) \boldsymbol{I}$ is the short-time averaged Lighthill stress tensor, $\tilde{\boldsymbol{t}}=-\tilde{\rho} \widetilde{\boldsymbol{u}^{\prime \prime} \boldsymbol{u}^{\prime \prime}}$ being the short-time averaged Reynolds stress tensor. This is derived following the procedure of Lighthill [28] from the short-time averaged Navier-Stokes equations and is exact.

The above inhomogeneous wave equation separates the linear acoustic field in the ambient medium at rest on the left hand side of the equation from the nonlinear acoustic sources arising from the unsteady flow. The solution procedure of Ffowcs Williams and Hawkings is used to integrate equation (11) over the cavity domain (Zhang et al. [10]). The acoustic density fluctuation perceived by an observer at $\boldsymbol{x}$ at time $t$ due to a finite volume $V(\boldsymbol{y})$ of unsteady flow emitting at the earlier time $\tau$ is

$$
\begin{aligned}
\rho(\boldsymbol{x}, t)= & \frac{1}{4 \pi c_{\infty}^{2}} \int_{V} \nabla \cdot \nabla \cdot \tilde{\boldsymbol{T}}(\boldsymbol{y}, \tau) \frac{d V(\boldsymbol{y})}{R^{*}} \\
& +\frac{1}{4 \pi} \oint_{S}\left(\nabla \tilde{\rho}+\frac{\tilde{\rho}}{R} \nabla R+\frac{\nabla \cdot \tilde{\rho} \tilde{\boldsymbol{u}}}{c_{\infty}} \nabla R\right) \cdot \boldsymbol{n} \frac{d S(\boldsymbol{y})}{R^{*}}
\end{aligned}
$$

where $t=\tau+R / c_{\infty}$ is the retarded or reception time, $R=|\boldsymbol{x}-\boldsymbol{y}|$ is the distance source-observer and $R^{*}=|\boldsymbol{x}-\boldsymbol{y}|-\boldsymbol{M}_{\infty} \cdot(\boldsymbol{x}-\boldsymbol{y})$ is the geometric scaling factor for acoustic propagation. The surface $S$ bounds the flow domain $V$ and $\boldsymbol{n}$ is its outward surface normal unit vector. 
In equation (12), $R^{*}$ accounts for the convection of the acoustic waves by a constant Mach number $\boldsymbol{M}_{\infty}$ that approximately models the flow above the enclosure boundary layer for the purpose of acoustic convection. Other flowacoustic interactions within the unsteady flow have not been modelled.

The volume integral in equation (12) operates on the second order Lighthill stress tensor differential that represents the aerodynamic sound sources from shearing flow. Specifically, noise is produced by momentum flux accelerations in the direction of the far-field observer at $\boldsymbol{x}$. These acoustic quadrupoles were shown by Lighthill [28] to radiate with an acoustic intensity $\propto U_{\infty}^{8} / c_{\infty}^{5}$. The surface integral term, evaluated along the cavity solid boundary, models the contributions to noise by the aerodynamic flow interacting with the enclosure walls. This first order differential term is a dipole noise source model that was shown by Curle [29] to give an acoustic intensity $\propto U_{\infty}^{6} / c_{\infty}^{3}$. At subsonic speeds, the dipole term would be expected to be the dominant contribution to noise. At the model flow regime, $M_{\infty}>1$, the dipole and quadrupole type contributions would be expected to be similar in magnitude and this study will focus on the quadrupole type noise sources from $\tilde{\boldsymbol{T}}$.

The fluctuations in $\tilde{\boldsymbol{T}}$ are estimated from the numerical database of the timedependent cavity aerodynamic model. High values of the root-mean-square value of $\tilde{\boldsymbol{T}}$ in the cavity flow identify the most acoustically active flow regions. Estimates of $\tilde{\boldsymbol{T}}_{r m s}$ are performed between the onset of the self-sustained oscillation and $\tilde{\tau} \sim \mathbf{O}(150)$. 


\section{Time Mean Flow}

At $M_{\infty}=1.5$, the main time averaged flow features are confined to the enclosure and to its immediate surroundings. A complex shock containing flow pattern develops above the enclosure (Fig. 3). The oblique shock above the upstream cavity edge forms a Mach angle $\left(\arcsin \left(1 / M_{\infty}\right)\right)$ with the streamwise direction. There are no appreciable shock distortions or reflections close to the top computational boundary $b 8$.

An unsteady shear layer forms downstream of the upstream cavity edge. Its time averaged centreline position, defined as $y_{2}$ at which $\bar{u}_{1}=0.5$, varies in the streamwise direction. The time averaged density local minimum ahead of the cavity downstream edge indicates the maximum in momentum transfer normal to the cavity open top surface. At the cavity downstream edge, the contour packing indicates mean flow impingement on the forward facing wall close to $y_{2}=0$.

The time averaged axial velocity contours in Fig. 4(a) and 4(b) indicate a varying shear layer growth rate in the streamwise direction. Within $1 D$ from the upstream cavity edge the shear layer develops as a free shear layer. Further downstream its growth rate is increased due to the presence of a stationary vortex at about $y_{1}=2 D$ inside the cavity. The vortex streamwise location is indicated by the maximum in the back flow velocity $\left(-\bar{u}_{1}\right)$ close to the cavity floor. The presence of the solid walls limits the mass entrainment in the sheared flow which is displaced outwards and the shear layer growth is reduced. The $\bar{u}_{1}=0$ contour starting at the upstream cavity edge re-attaches on the downstream wall close to $y_{2}=0$, indicating a stagnation point. The 
time averaged displacement thickness $\left(\bar{\delta}_{1}\right)$ and momentum thickness $\left(\bar{\delta}_{2}\right)$ of the re-attached flow above the downstream cavity edge are about twice the upstream values of $0.857 \mathrm{~mm}$ and $0.384 \mathrm{~mm}$. The result from using the $320 \times 400$ computational mesh shown in Fig. 4(b) indicate that the time averaged flow prediction is almost grid independent.

The time averaged pressure on the upstream wall (Fig. 5) is predicted as a nearly constant value of $\bar{p}_{w} \sim 1.11 p_{\infty}$. On the cavity floor a local minimum is reached at about $y_{1}=2 D$, below the stationary vortex. The time averaged wall pressure than increases monotonically towards the downstream bottom cavity corner, where the fluid is nearly at rest $\left(\bar{p}_{w}=1.32 p_{\infty}\right)$. On the downstream wall, $\bar{p}_{w}$ has two maxima at $y_{2}=-1.0 D$ and $y_{2} \sim 0.0 D$, where the flow stagnates and $\bar{p}_{w}=1.32 p_{\infty}$ at both locations.

At $M_{\infty}=2.5$ a re-compression shock exists ahead of at the cavity downstream edge (Fig. 6). Its intensity is higher than at $M_{\infty}=1.5$. The shear layer time averaged centreline position is more constant in the streamwise direction than at the lower Mach number. This suggests a more uniform shear layer growth up to $y_{1}=2 D$, which is confirmed by the time averaged axial velocity predictions in Fig. 7. The displacement and momentum thickness above the downstream cavity edge are approximately 1.5 times the upstream values of $1.353 \mathrm{~mm}$ and $0.332 \mathrm{~mm}$.

The time averaged pressure around the cavity perimeter in Fig. 8 shows a trend similar to the prediction at $M_{\infty}=1.5$. The time averaged pressure maximum at the cavity downstream edge (stagnation point) is more pronounced, due to the higher free stream Mach number. Similar results are given in Jeng \& Payne [9] and in Zhang et al. [14], showing similar levels of agreement with 
the experiments of Zhang [6].

The measured time averaged cavity pressure drag coefficients $\left(C_{D}\right)$ are 0.049 and 0.037 at $M_{\infty}=1.5$ and $M_{\infty}=2.5$ respectively. The predicted values are 0.055 and 0.030 . The agreement is generally good.

\section{Unsteady Flow}

\subsection{Self-excited instability}

The unsteady open cavity flow at $M_{\infty}=1.5$ is visualized in a selection of numerical interferograms/contours in Figs. 9(b) \& (d). The phase-matched result from holographic interferometry by Zhang [6] is given for comparison in Figs. 9(a) \& (c).

The in-coming turbulent boundary layer separates at the upstream cavity edge, forming a free shear layer. This is responsive to the unsteady pressure and vorticity field inside the enclosure and develops Kelvin-Helmholtz type instabilities. The unsteady modes saturate within a short distance from the upstream cavity edge and a rolled-up vortex is formed as shown in Figs. 9(a) \& (b). The vortex is convected downstream by the shear flow.

The upstream cavity edge shock is unsteady and its location and strength are

affected by the shear layer normal displacement beneath it [30]. When the shear layer is displaced in the positive normal direction, the shock is displaced upstream and has greater strength as shown in Figs. 9(c) \& (d). Conversely the shock strength decreases when the shear layer displaces towards the cavity floor. 
Mass and momentum exchange between the free stream and the enclosed flow occurs across the open cavity top boundary. Unsteady mass ejection and entrainment at the downstream cavity edge are highlighted in Figs. 9(a) \& (b) and Figs. 9(c) \& (d) respectively. During the mass ejection phase, a vortex leaves the cavity and is convected downstream, parallel to the free stream. The convected vorticity leaves the computational domain without producing noticeable vorticity wave reflections in the subsonic section of the boundary layer.

The interaction of the unsteady velocity and vorticity fields at the downstream cavity edge generates an excess unsteady pressure field within the cavity and acts on the shear layer at the upstream edge, exciting it and providing the feed-back to maintain self-sustained flow oscillations. The numerical results indicate that the numerical model has captured this essential time-dependent feature of the unsteady flow.

At $M_{\infty}=2.5$ the shear layer flow-normal oscillation is the most prominent flow feature (Fig. 10). This drives the self-sustained flow instability. A complex shock pattern develops above the enclosure, which is dependent on the shear layer vertical displacement. These transient compressible flow features can be explained by analogy with a supersonic flow past a wavy wall. At $M_{\infty}=2.5$ the dominant mode phase speed is approximately $\mathbf{O}\left(0.5 U_{\infty}\right)$, therefore giving a relative flow Mach number of approximately 1.25. Regions of shear layer maximum concavity display compressive shocks with a $(\pi / 4)$ Mach angle, close to the shear layer. This indicates a $\tilde{M}=1.5$ local Mach number. Expansion waves are generated above regions of shear layer maximum convexity. Unsteady mass entrainment and ejection occurs also at $M_{\infty}=2.5$, the phase sequence in Figs. 9 and 10 being identical. Convected vortical structures are 
generated at the downstream cavity edge by shear layer 'clipping' at the end of the mass ejection phase (Neary \& Stephanoff [31]).

\subsection{Pressure fluctuation}

The self-sustained characteristic of the flow instability is evident in the normalized short-time averaged wall pressure history predictions (Figs. 11 and 12). Self-sustained oscillations are obtained after a $\tilde{\tau}=50$ initial period during which the starting transients are allowed to decay below the numerical noise level. The instability is dominated by phase-locked cavity modes of constant amplitude. At $M_{\infty}=1.5$ the pressure power spectral density measured at $(2.33 D,-1 D)$ peaks at Strouhal numbers $(S t r)$ 0.093, 0.208 (dominant mode), and 0.323. At $M_{\infty}=2.5$ the corresponding values are 0.092, 0.179, and 0.264 (dominant mode). At the same location, the predicted values are $0.094,0.192$, and 0.369 at $M_{\infty}=1.5$ and $0.090,0.176$, and 0.262 at $M_{\infty}=2.5$. The agreement is generally good.

The pressure history predicted by the numerical method at $(0.33 D,-1 D)$, $(2.33 D,-1 D),(5.66 D, 0 D)$, and $(7.66 D, 0 D)$ indicates that the fluctuation of largest amplitude occurs inside the enclosure in the neighbourhood of the second transducer $(2.33 D,-1 D)$, close to the downstream wall. A more accurate quantification of the unsteady pressure loading on the cavity perimeter is performed by estimating the sound pressure level (SPL) along the walls (Fig. 13). The highest levels are predicted at the downstream cavity corner and edge and are $\sim 182 \mathrm{~dB}$ re $20 \mu \mathrm{Pa}$ at $M_{\infty}=1.5$. This is higher but still comparable with $171.5 \mathrm{~dB}$ measured by Zhang [7] at $(2.33 D,-1 D)$. At these locations, the unsteady mass impingement and ejection results in alternating phases of 
high speed flow past the edge and of shear flow stagnating on the downstream wall. At the downstream corner, the unsteady recirculation is responsible for the predicted pressure unsteadiness. The downstream wall is clearly a region of significant flow unsteadiness in the cavity aerodynamic field. The shear flow interaction with the geometry, resulting in the observed unsteady mass and momentum exchange between the enclosure and the free stream, is the driving mechanism for the unsteady aerodynamic pressure field.

A second $S P L$ maximum of $\sim 178 \mathrm{~dB}$ re $20 \mu \mathrm{Pa}$ is predicted at the upstream cavity edge. The fluctuation amplitude is adequate to locally excite the shear layer, triggering convectively amplified modes at the observed Strouhal numbers. The predicted unsteady aerodynamic pressure is therefore the link to close the feed-back loop to maintain the self-excited flow instability. On the upstream bulkhead $\left(y_{1}<0\right)$ the upstream propagating disturbances are confined to the boundary layer, below the sonic line. Consequently, the wall pressure fluctuation is damped in the upstream direction, its magnitude being reduced below $120 \mathrm{~dB}$ re $20 \mu \mathrm{Pa}$ by $y_{1} \sim-0.6 D$. At $M_{\infty}=2.5$ the numerical method predicts the same trend for wall $S P L$ as at the lower Mach number, the fluctuation amplitude being $\mathbf{O}(10 \mathrm{~dB}$ re $20 \mu \mathrm{Pa})$ lower throughout. These results are in general agreement with the numerical predictions by Jeng \& Payne [9].

\subsection{Aerodynamic noise sources}

The physics of aerodynamic noise production from compressible turbulent nonuniform flows is complex. In this study, some characteristics of this process are disclosed, which relate to the large-scale flow instability. 
The unsteady pressure predictions highlight the flow region at the downstream cavity edge as potentially the most unsteady. The accelerating fluid in the direction of the observer produces aerodynamic sound, as described by the application of the Lighthill solution by Proudman [32]. Estimating the rootmean-square of the streamwise and normal flow velocities therefore gives some description of the noise production physics at the downstream cavity edge.

The prediction at $M_{\infty}=1.5$ in Fig. 14(a) shows that $\left(\tilde{u}_{1}\right)_{r m s}$, normalized as in Section 2, is maximum at the downstream cavity edge and is 0.272 . The region of maximum $\left(\tilde{u}_{1}\right)_{r m s}$ extends mainly in the streamwise direction. This suggests that a noise source of streamwise longitudinal and lateral quadrupole type is present at this location in the aerodynamic field. A second localized $\left(\tilde{u}_{1}\right)_{r m s}$ maximum is located at the upstream edge.

The root-mean-square normal velocity near-field is mainly related to the shear layer normal displacement. Two regions of maximum $\left(\tilde{u}_{2}\right)_{r m s}$ are shown in Fig. 14(b) along the $y_{2}=0$ line at approximately $0.5 D$ and $2 D$ from the upstream edge. The region closest to the upstream edge is where a vortex is periodically rolled up. This region is localized and $\left(\tilde{u}_{2}\right)_{r m s}$ decays rapidly with distance from its point of maximum value. The other region occurs where the shear layer normal displacement is maximum. These indicate that noise sources of the normal longitudinal quadrupole type are present at $(0.5 D, 0 D)$ and $(2 D, 0 D)$ in the aerodynamic field. Their strength are expected to be less then the streamwise longitudinal quadrupole sources, since the associated $\left(\tilde{u}_{2}\right)_{r m s}$ maximum is 0.175 .

The unsteadiness in the $M_{\infty}=2.5$ flow (Fig. 15) is proportionally lower than at $M_{\infty}=1.5$, as also indicated by the $S P L$ predictions in Section 5.2. At the 
higher Mach number, a $\left(\tilde{u}_{1}\right)_{r m s}$ maximum of 0.223 is located at approximately $y_{1}=2 D$ on the $y_{2}=0$ line. The surrounding region of high $\left(\tilde{u}_{1}\right)_{r m s}$ is elongated in the streamwise direction and results from the combined effect of the periodic shear layer oscillation and of a normal velocity gradient being present across the shear layer. A localized maximum is also visible above the downstream edge and is related to the shear layer clipping and periodic vortex shedding. The $\left(\tilde{u}_{2}\right)_{r m s}$ contours also indicate a maximum at this location, highlighting this region as an important noise source also at $M_{\infty}=2.5$.

The time averaged characteristics of the aerodynamic noise source near-field are now considered. The short-time root-mean-square Lighthill stress tensor $\left(\tilde{\boldsymbol{T}}_{r m s}\right)$ is estimated in the computational domain (Figs. 16 and 17).

At both Mach numbers, the contributions from $\tilde{T}_{11}$ to far-field cavity noise are dominant, which is what would be expected from a predominantly longitudinal oscillation. According to Pierce [33], this streamwise longitudinal quadrupole type noise source is expected to give a $\cos ^{2} \theta$ far-field directivity pattern, modified by the effects of source convection. The highest values occur close to the downstream cavity edge, in agreement with the $\left(\tilde{u}_{1}\right)_{r m s}$ predictions. The nature of this noise source is also confirmed, this being a momentum flux fluctuation $(\tilde{\rho} \tilde{\boldsymbol{u}} \tilde{\boldsymbol{u}})$ in the direction of the observer. The physical process generating such fluctuation is associated with the periodic mass entrainment and ejection occurring at this location. Another area of high $\left(\tilde{T}_{11}\right)_{r m s}$ is near the upstream cavity edge and is probably associated to the fluctuations in momentum flux during the vortex roll up. The fluid flow entrainment increases the shear layer thickness, producing a localized $\left(\tilde{T}_{11}\right)_{r m s}$.

The lateral quadrupole type source contribution to the far-field noise is gen- 
erated in the shear layer (Figs. 16(b) and 17(b)). At $M_{\infty}=1.5$, the maximum $\left(\tilde{T}_{12}\right)_{r m s}$ is 0.130 . This is located close to the upstream cavity edge where a localized large $\left(\tilde{u}_{2}\right)_{r m s}$ was identified in Fig. 14(b). Here the fluctuations in fluid momentum flux are driven by the unsteady vorticity field which is characterized by vortex roll up. The convected vortex generates a moving bow shock above the cavity the intensity of which decreases in the streamwise direction, while the shear layer thickens. This limits the area of high $\left(\tilde{T}_{12}\right)_{r m s}$ to the initial portion of the shear layer, $0 D \leq y_{1} \leq 1 D$. At $M_{\infty}=2.5$, the observed instability mode does not involve regular vortex shedding from the upstream cavity edge and a region of high $\left(\tilde{T}_{12}\right)_{r m s}$ is where the shear layer displacement is maximum. The interaction between the unsteady shear layer and the downstream cavity edge is responsible for the localized $\left(\tilde{T}_{12}\right)_{r m s}$ maximum.

Minor contributions to far-field noise are from aeroacoustic sources of the transverse longitudinal quadrupole type, as shown in Figs. 16(c) and 17(c). These are generated by the unsteady flow in the neighbourhood of the normal downstream cavity wall where fluid is periodically accelerated into the clockwise cavity flow recirculation.

All regions of high $\left(\tilde{\boldsymbol{T}}_{r m s}\right)$ are located inside the cavity or in its immediate surroundings, clear from all open flow boundaries (Figs. 16 and 17). Around the open flow boundary perimeter, $\left(\tilde{\boldsymbol{T}}_{r m s}\right)$ is largest in the subsonic part of the boundary layer at the outflow, where convected vortices leave the computational domain. At $M_{\infty}=1.5$, the average $\left(\tilde{T}_{11}\right)_{r m s}$ across the boundary layer is 0.06 of the near-field maximum. At $M_{\infty}=2.5$, it is 0.03. Similar results are produced for the lateral and normal longitudinal quadrupole source fields. This confirms that the computational domain size selected for this study covers the most acoustically active flow regions for the selected test cases. 


\section{Conclusions}

A numerical investigation was performed on the time-mean and time-dependent characteristics of supersonic open cavity flows at $M_{\infty}=1.5$ and $M_{\infty}=2.5$. An enhanced computational fluid dynamic method was adopted to further the analysis by Zhang [7] and address cavity noise generation.

The time-mean flow predictions highlighted the importance of the stationary vortex at $y_{1} \sim 2 D$ inside the cavity in determining the drag coefficient. The low pressure in the vortex core displaces the shear layer towards the enclosure halfdepth, entraining mass and momentum and increasing the time-mean shear layer thickness. The entrained momentum is responsible for the time-mean high pressure on the downstream wall, giving positive time-mean drag. The wall pressure distribution was found in good agreement with measurements at both Mach numbers. Zhang et al. [13] show that significant reductions in $C_{D}$ can be achieved when the downstream cavity corner is modified by a ramp and the stationary vortex is suppressed.

The time-mean growth rate of the shear layer and its normal displacement along the cavity open boundary vary in the streamwise direction, leading to a complex wave pattern above the enclosure. These time-mean streamwise shear layer oscillations might lead to a variable phase velocity and convective non-linearities in the downstream propagating instability modes, affecting the dominant mode Strouhal number and its kinetic energy transfer to higher harmonics. Further analysis is required on this topic.

On time-dependent flow, the model reproduces the essential physics of the large-scale structure observed in experiment. At $M_{\infty}=1.5$, self-sustained 
shear layer oscillations combine with periodic vortex shedding from the cavity leading edge. The interaction of the convected instabilities with the downstream cavity corner results in alternating phases of localized mass impingement and ejection that are visualized in the compressible flow. At $M_{\infty}=2.5$, vortex free shear layer oscillations are predicted, in agreement with experiment.

An unsteady aerodynamic pressure field in the cavity is predicted in the numerical simulation. The physical mechanism generating aerodynamic pressure fluctuations is addressed and the downstream cavity edge is identified as a critical area of aerodynamic pressure and flow instability. The predicted $S P L$ at this location is $\sim 182 \mathrm{~dB}$ re $20 \mu \mathrm{Pa}$ at $M_{\infty}=1.5$. This is the highest level of unsteady aerodynamic pressure loading predicted around the enclosure perimeter and it could represent a design constraint in practical engineering applications.

At the upstream cavity edge, the unsteady pressure perturbs the shear layer, closing the feed-back loop for phase-locked self-sustained oscillations. Here the predicted $S P L \sim 178 \mathrm{~dB}$ re $20 \mu \mathrm{Pa}$ at $M_{\infty}=1.5$ is of the right order of magnitude to provide such interaction between the aerodynamic pressure field and the shear flow.

The aeroacoustic investigation focused on (i) the location and quantification of the sources of aerodynamic noise in the cavity flow and (ii) addressing the physics of noise production to improve its current understanding towards its suppression.

The cavity flow was found to be an acoustically compact source in that all regions of large contributions to the acoustic radiation are located inside the enclosure or in its immediate surroundings. The unsteady shear layer plays 
a dominant role in the acoustic radiation: the momentum flux fluctuation due to the self-sustained instability is the dominant cavity noise generating mechanism. Longitudinal quadrupole type sources were predicted along the shear layer which reflect the aerodynamic fluctuation being predominantly of the longitudinal type.

Areas of high acoustic source strength $\left(\tilde{\boldsymbol{T}}_{r m s}\right)$ are the neighbourhood of the cavity edges. The vortex roll up near the upstream edge and the unsteady mass exchange near the downstream edge provide the fluctuations in momentum flux for noise generation. Thus, modifications to the edge geometry could prove effective to reduce both cavity drag and the radiated noise.

\section{Acknowledgements}

The author wishes to thank Prof. X. Zhang and Prof. G. M. Lilley, University of Southampton, UK, for their helpful guidance in the preparation of this article, and Prof. J. A. Edwards for supporting the use of the CFD software. This work was undertaken at the University of Southampton as part of the author's PhD and was supported by EPSRC grant GR-L-08014.

\section{Nomenclature}

c Speed of sound

$C_{D} \quad$ Drag coefficient, $(2 / D) \int_{0}^{D}\left(\left.\tilde{p}_{w}\right|_{y_{1}=3}-\left.\tilde{p}_{w}\right|_{y_{1}=0}\right) d y_{2}$

e $\quad$ Specific internal energy

I Identity matrix 
$u_{1} \quad$ Streamwise velocity

$u_{2} \quad$ Normal velocity

$t \quad$ Retarded time

$\boldsymbol{t} \quad$ Turbulent stress tensor

$T_{i j} \quad i j^{\text {th }}$ component of Lighthill's stress tensor

$\boldsymbol{T} \quad$ Lighthill's stress tensor

$\boldsymbol{U}$ Conservative variable vector

$y_{1} \quad$ Streamwise coordinate

$y_{2} \quad$ Normal coordinate

$\gamma \quad$ Ratio of specific heats, $C_{p} / C_{\mathrm{V}}$

$\mu_{l} \quad$ Dynamic viscosity

$\mu_{t} \quad$ Eddy viscosity

$\omega \quad$ Specific dissipation rate of turbulent kinetic energy

$\tau \quad$ Time

$\tau \quad$ Viscous stress tensor

$\square^{2} \quad$ Wave operator, $\square^{2}=\partial^{2} / \partial t^{2}-c_{\infty}^{2} \nabla^{2}$

()$_{i} \quad$ Inviscid component

()$_{r m s}$ Root-mean-square value

( ) stagnation value

()$^{T} \quad$ Transpose operator

()$_{t} \quad$ Turbulent component

()$_{w}$ Wall boundary value

()$_{\infty}$ Free stream value

() Non-dimensional short-time averaged value 
() Non-dimensional time averaged value

\section{References}

[1] C. Rowley, T. Colonius, A. Basu, On self-sustained oscillations in twodimensional compressible flow over rectangular cavities, Journal of Fluid Mechanics 455 (2) (2002) 315-346.

[2] D. Rockwell, E. Naudascher, Review - Self-sustaining oscillations of flow past cavities, Transactions of the American Society of Mechanical Engineers, Journal of Fluids Engineering 100 (1978) 152-165.

[3] W. K. Blake, A. Powell, The development of contemporary views of flowtone generation, in: A. Krothapalli, C. A. Smith (Eds.), Recent advances in aeroacoustics: proceedings of an international symposium held at Stanford University, August 22-26, 1986, Springer-Verlag, 1986, pp. 247-345.

[4] T. Colonius, An overview of simulation, modeling, and active control of flow/acoustic resonance in open cavities, Conference Paper 2001-0076, AIAA, 39th AIAA Aerospace Sciences Meeting \& Exhibit, Reno, NV, USA (Jan. 2001).

[5] V. Theofilis, Advances in global linear instability analysis of nonparallel and three-dimensional flows, Progress in Aerospace Sciences 39 (4) (2003) 249-315.

[6] X. Zhang, An experimental and computational investigation into supersonic shear layer driven single and multiple cavity flowfields, Ph.D. thesis, Department of Engineering, University of Cambridge (Feb. 1988).

[7] X. Zhang, Compressible cavity flow oscillation due to shear layer instabilities and pressure feedback, AIAA Journal 33 (8) (1995) 1404-1411. 
[8] A. F. Charwat, J. N. Roos, F. C. J. Dewey, J. A. Hitz, An investigation of separated flows - Part I: The pressure field, Journal of the Aerospace Sciences 28 (1961) 457-470.

[9] Y. Jeng, U. Payne, Numerical study of a supersonic open cavity flow and pressure oscillation control, Journal of Aircraft 32 (2) (1995) 363-369.

[10] X. Zhang, A. Rona, G. Lilley, Far-field noise radiation from an unsteady supersonic cavity flow, in: Proceedings of the First Joint CEAS/AIAA Aeroacoustics Conference, Munich, 1995, pp. 285-292.

[11] S. Grace, An overview of computational aeroacoustic techniques applied to cavity noise prediction, Conference Paper 2001-510, AIAA, 39th AIAA Aerospace Sciences Meeting \& Exhibit, Reno, NV, USA (Jan. 2001).

[12] C. W. Rowley, D. R. Williams, Dynamics and control of high-reynolds-number flow over open cavities, Annual Review of Fluid Mechanics 38 (2006) 251-276.

[13] X. Zhang, A. Rona, J. A. Edwards, The effect of trailing edge geometry on cavity flow oscillation driven by a supersonic shear layer, The Aeronautical Journal 102 (1013) (1998) 129-136.

[14] X. Zhang, X. X. Chen, A. Rona, J. A. Edwards, Attenuation of cavity flow oscillation through leading edge flow control, Journal of Sound and Vibration 221 (1) (1999) 23-47.

[15] P. Block, Noise response of cavities of varying dimensions at subsonic speeds, Technical Note D-8351, NASA (Dec. 1976).

[16] J. C. Hardin, D. S. Pope, An acoustic/viscous splitting technique for computational aeroacoustics, Theoretical and Computational Fluid Dynamics 6 (5-6) (1994) 323-340.

[17] A. J. Bilanin, E. E. Covert, Estimation of possible excitation frequencies for shallow rectangular cavities, AIAA Journal 11 (3) (1973) 347-351. 
[18] C. K. W. Tam, P. J. Block, On the tones and pressure oscillations induced by flow over rectangular cavities, Journal of Fluid Mechanics 89 (2) (1978) 373-399.

[19] X. Zhang, J. Edwards, Computational analysis of unsteady supersonic cavity flow driven by thick shear layers, Aeronautical Journal 92 (919) (1988) 365-374.

[20] A. Rona, W. Dieudonné, Unsteady laminar and turbulent cavity flow models by second order upwind methods, Conference Paper 99-0656, AIAA, 37th AIAA Aerospace Sciences Meeting \& Exhibit, Reno, NV, USA (Jan. 1999).

[21] D. Wilcox, Turbulence modeling for CFD, DCW Industries Inc., La Cãnada, California, USA, 1993.

[22] D. Mabey, W. Sawyer, Experimental studies of the boundary layer on a flat plate at Mach numbers from 2.5 to 4.5, Reports and Memoranda 3784, British Aeronautical Research Council (Sep. 1974).

[23] G. Lilley, X. Zhang, A. Rona, Progress in computational aeroacoustics in predicting the noise radiated from turbulent flows, International Journal of Acoustics and Vibration 2 (1) (1997) 3-10.

[24] P. Roe, Approximate Riemann solvers, parameter vectors, and difference schemes, Journal of Computational Physics 43 (1981) 357-372.

[25] A. Rona, Aerodynamic and aeroacoustic estimations of oscillatory supersonic flows, Ph.D. thesis, Department of Aeronautics and Astronautics, University of Southampton, UK (Jun. 1997).

[26] M. Manna, A three dimensional high resolution compressible flow solver, Ph.D. thesis, Université Catholique de Louvain (Oct. 1992).

[27] F. Bastin, Etude numérique du bruit de jet rayonné par les structures cohérentes de la turbulence, Report EDF-DER-96-NV-00015, Electricité de France, Direction des études et recherches, Clamart, France (1996). 
[28] M. Lighthill, On sound generated aerodynamically: I. General theory, Proceedings of the Royal Society of London, Series A, 211 (1107) (1952) 564587.

[29] N. Curle, The influence of solid boundaries upon aerodynamic sound, Proceedings of the Royal Society of London, Series A, 231 (1187) (1955) 505514.

[30] X. Zhang, A. Rona, J. A. Edwards, An observation of pressure waves around a shallow cavity, Journal of Sound and Vibration 214 (4) (1998) 771-778.

[31] M. Neary, K. Stephanoff, Shear-layer-driven transition in a rectangular cavity, Physics of Fluids 30 (10) (1987) 2936-2946.

[32] I. Proudman, The generation of noise by isotropic turbulence, Proceedings of the Royal Society of London, Series A, 214 (1116) (1952) 119-132.

[33] A. Pierce, Acoustics: An introduction to its physical principles and applications, McGraw-Hill, New York, 1981.

[34] X. Zhang, A. Rona, G. Lilley, Self-excited supersonic flow aeroacoustic: Aerodynamic field, in: Proceedings of the 10th International Conference on Numerical Methods for Laminar and Turbulent Flow, Swansea, UK, 1997, pp. $1-29$.

\section{List of Figures}

1 Shear layer driven cavity flow computational domain.

2 Schematic of the initial cavity flow conditions. 
$3 M_{\infty}=1.5$ cavity flow. Time averaged density contours, $\bar{\rho}_{\min }=0.67, \bar{\rho}_{\max }=1.12, \Delta \bar{\rho}=0.03$. (a) $160 \times 200$ computational mesh, (b) $320 \times 400$ computational mesh.

$4 \quad M_{\infty}=1.5$ cavity flow. Time averaged streamwise velocity contours, $\left(\bar{u}_{1}\right)_{\min }=-0.32,\left(\bar{u}_{1}\right)_{\max }=1.02, \Delta \bar{u}_{1}=0.08$. (a) $160 \times 200$ computational mesh, (b) $320 \times 400$ computational mesh.

$5 \quad M_{\infty}=1.5$ cavity flow time averaged wall pressure. (a) approaching surface, (b) upstream wall, (c) floor, (d) downstream wall. (-) computation, (o) measurements [7].

$6 M_{\infty}=2.5$ cavity flow. Time averaged density contours, $\bar{\rho}_{\text {min }}=0.42, \bar{\rho}_{\max }=1.10, \Delta \bar{\rho}=0.03$.

$7 \quad M_{\infty}=2.5$ cavity flow. Time averaged axial velocity contours, $\left(\bar{u}_{1}\right)_{\min }=-0.31,\left(\bar{u}_{1}\right)_{\max }=1.01, \Delta \bar{u}_{1}=0.08$.

$8 \quad M_{\infty}=2.5$ cavity flow time averaged pressure. (a) approaching surface, (b) upstream wall, (c) floor, (d) downstream wall. (-) computation, (o) measurement [7].

$9 \quad M_{\infty}=1.5$ cavity holographic interferograms. Mass ejection [34] (a) and prediction (b), mass entrainment [23] (c) and prediction (d). $\Delta \tilde{\rho}=0.03$.

$10 M_{\infty}=2.5$ cavity holographic interferograms. Mass ejection [34] (a) and prediction (b), mass entrainment [34] (c) and prediction (d). $\Delta \tilde{\rho}=0.03$. 
$11 M_{\infty}=1.5$ short-time averaged pressure history.

$12 M_{\infty}=2.5$ short-time averaged pressure history.

13 Sound pressure level on the cavity walls. (-) $M_{\infty}=1.5,(--)$

$$
M_{\infty}=2.5 \text {. }
$$

$14 M_{\infty}=1.5$ cavity short-time root mean square velocity contours. (a) streamwise component, $\left[\left(\tilde{u}_{1}\right)_{r m s}\right]_{\max }=0.272,(\mathrm{~b})$ normal component, $\left[\left(\tilde{u}_{2}\right)_{r m s}\right]_{\max }=0.175 . \Delta \tilde{u}_{r m s}=0.03$.

$15 M_{\infty}=2.5$ cavity short-time root mean square velocity contours. (a) streamwise component, $\left[\left(\tilde{u}_{1}\right)_{r m s}\right]_{\max }=0.223$, (b) normal component, $\left[\left(\tilde{u}_{2}\right)_{r m s}\right]_{\max }=0.094 . \Delta \tilde{u}_{r m s}=0.03$.

$16 M_{\infty}=1.5$ cavity root mean square $\tilde{\boldsymbol{T}}$ contours. (a) $\left(\tilde{T}_{11}\right)_{r m s}$, $\left[\left(\tilde{T}_{11}\right)_{r m s}\right]_{\max }=0.480,(\mathrm{~b})\left(\tilde{T}_{12}\right)_{r m s},\left[\left(\tilde{T}_{12}\right)_{r \operatorname{ms}}\right]_{\max }=0.130$, (c) $\left(\tilde{T}_{22}\right)_{r m s},\left[\left(\tilde{T}_{22}\right)_{r m s}\right]_{\max }=0.083 . \Delta \tilde{\boldsymbol{T}}_{r m s}=0.01$.

$17 M_{\infty}=2.5$ cavity root mean square $\tilde{\boldsymbol{T}}$ contours. (a) $\left(\tilde{T}_{11}\right)_{r m s}$, $\left[\left(\tilde{T}_{11}\right)_{r m s}\right]_{\text {max }}=0.304,(\mathrm{~b})\left(\tilde{T}_{12}\right)_{r m s},\left[\left(\tilde{T}_{12}\right)_{r m s}\right]_{\max }=0.046$, (c) $\left(\tilde{T}_{22}\right)_{r m s},\left[\left(\tilde{T}_{22}\right)_{r m s}\right]_{\text {max }}=0.042 . \Delta \tilde{\boldsymbol{T}}_{r m s}=0.01$. 


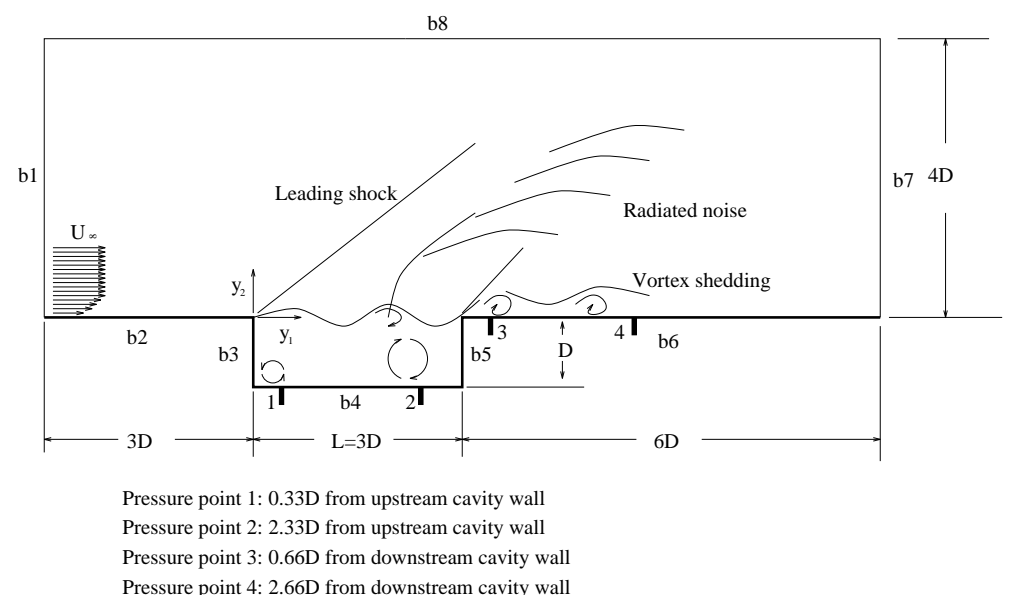

Fig. 1. Shear layer driven cavity flow computational domain.

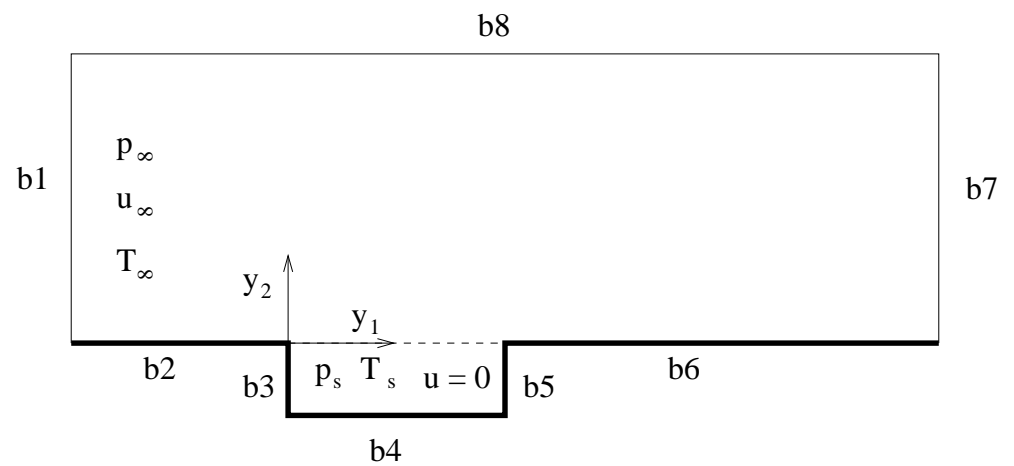

Fig. 2. Schematic of the initial cavity flow conditions. 

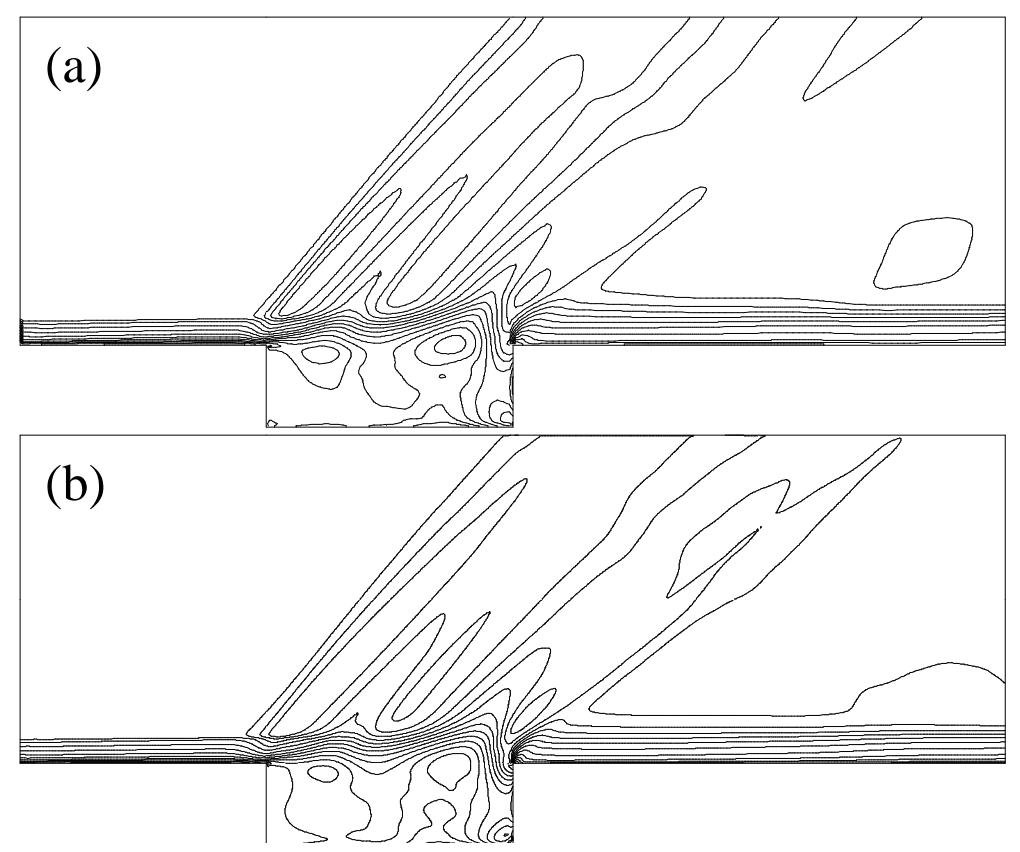

Fig. 3. $M_{\infty}=1.5$ cavity flow. Time averaged density contours, $\bar{\rho}_{\min }=0.67$, $\bar{\rho}_{\max }=1.12, \Delta \bar{\rho}=0.03$. (a) $160 \times 200$ computational mesh, (b) $320 \times 400$ computational mesh.

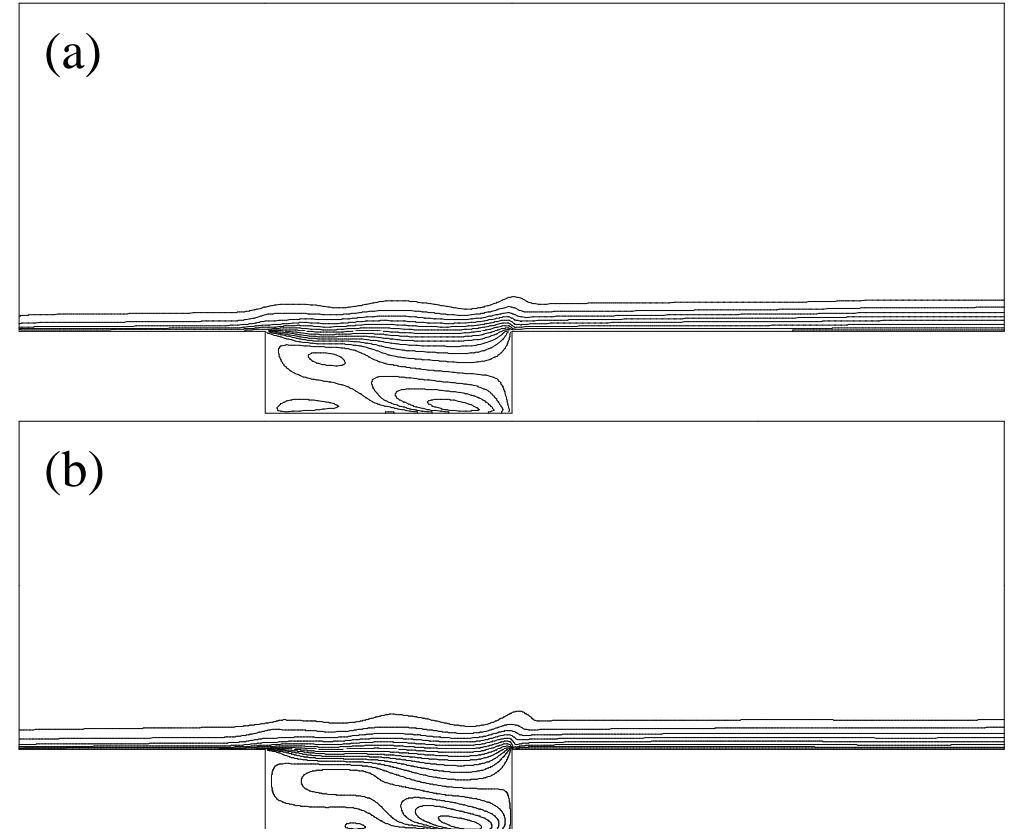

Fig. 4. $M_{\infty}=1.5$ cavity flow. Time averaged streamwise velocity contours, $\left(\bar{u}_{1}\right)_{\min }=-0.32,\left(\bar{u}_{1}\right)_{\max }=1.02, \Delta \bar{u}_{1}=0.08$. (a) $160 \times 200$ computational mesh, (b) $320 \times 400$ computational mesh. 

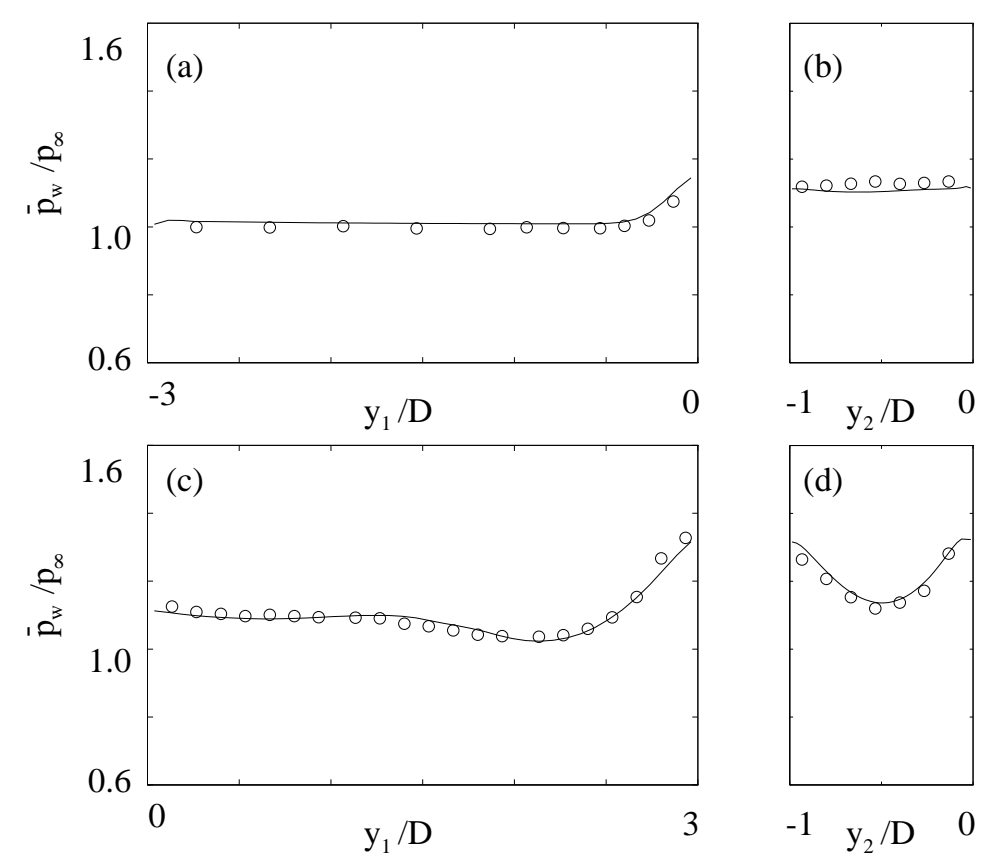

Fig. $5 . M_{\infty}=1.5$ cavity flow time averaged wall pressure. (a) approaching surface, (b) upstream wall, (c) floor, (d) downstream wall. (-) computation, (o) measurements [7].

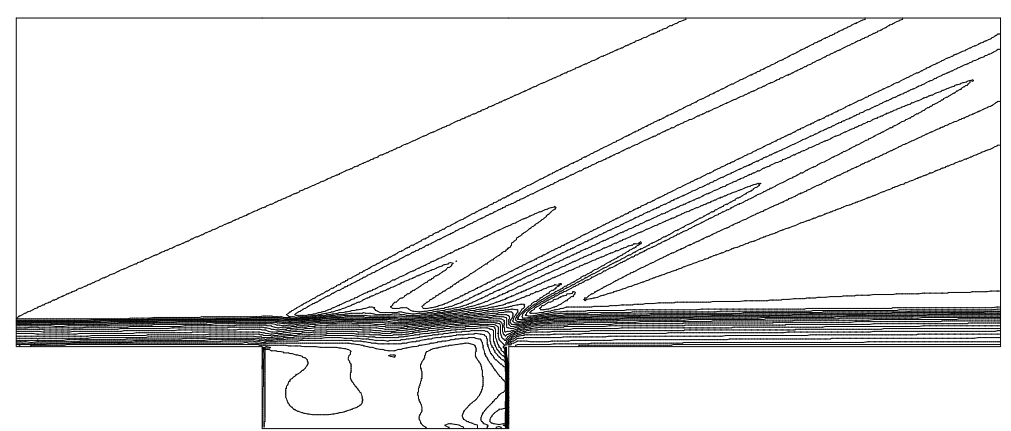

Fig. 6. $M_{\infty}=2.5$ cavity flow. Time averaged density contours, $\bar{\rho}_{\min }=0.42$, $\bar{\rho}_{\text {max }}=1.10, \Delta \bar{\rho}=0.03$. 


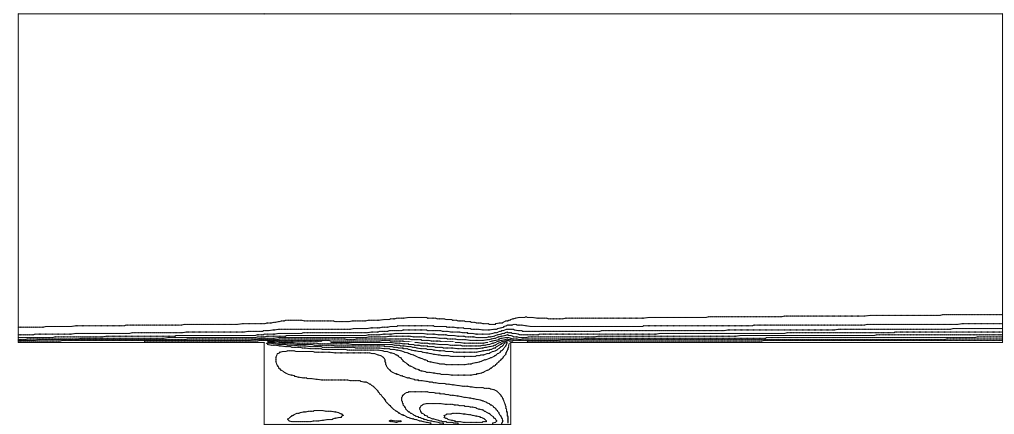

Fig. 7. $M_{\infty}=2.5$ cavity flow. Time averaged axial velocity contours, $\left(\bar{u}_{1}\right)_{\min }=-0.31,\left(\bar{u}_{1}\right)_{\max }=1.01, \Delta \bar{u}_{1}=0.08$.
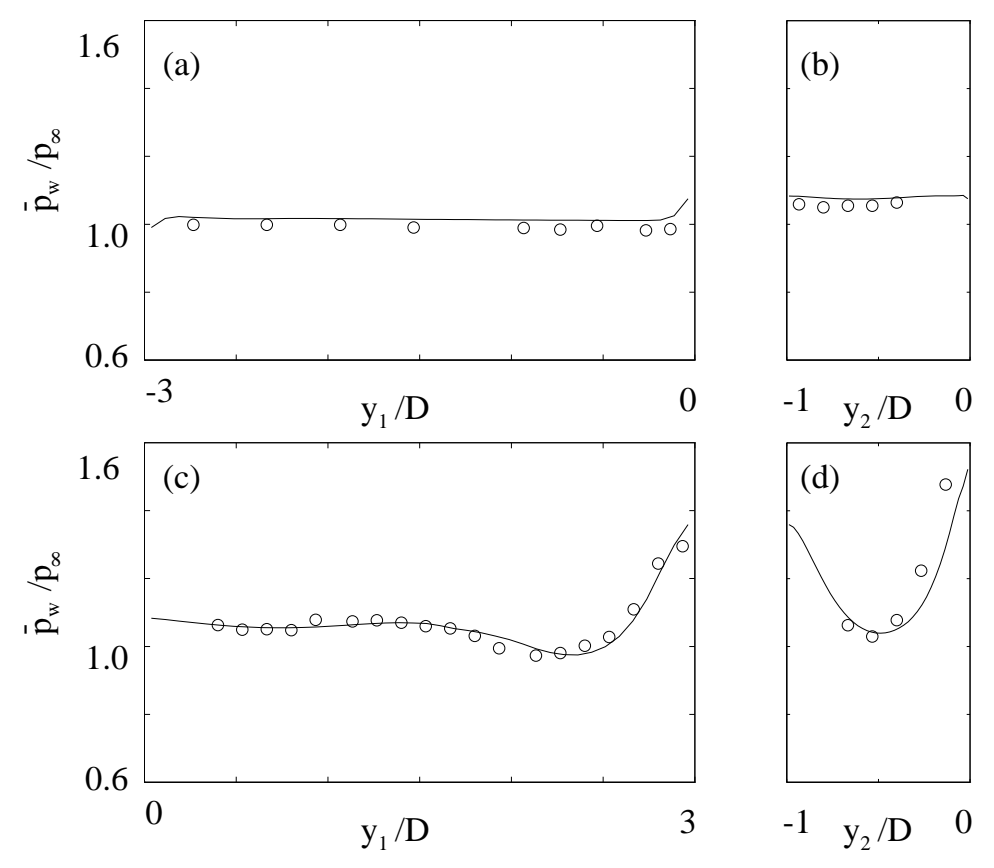

Fig. 8. $M_{\infty}=2.5$ cavity flow time averaged pressure. (a) approaching surface, (b) upstream wall, (c) floor, (d) downstream wall. (-) computation, (o) measurement [7]. 


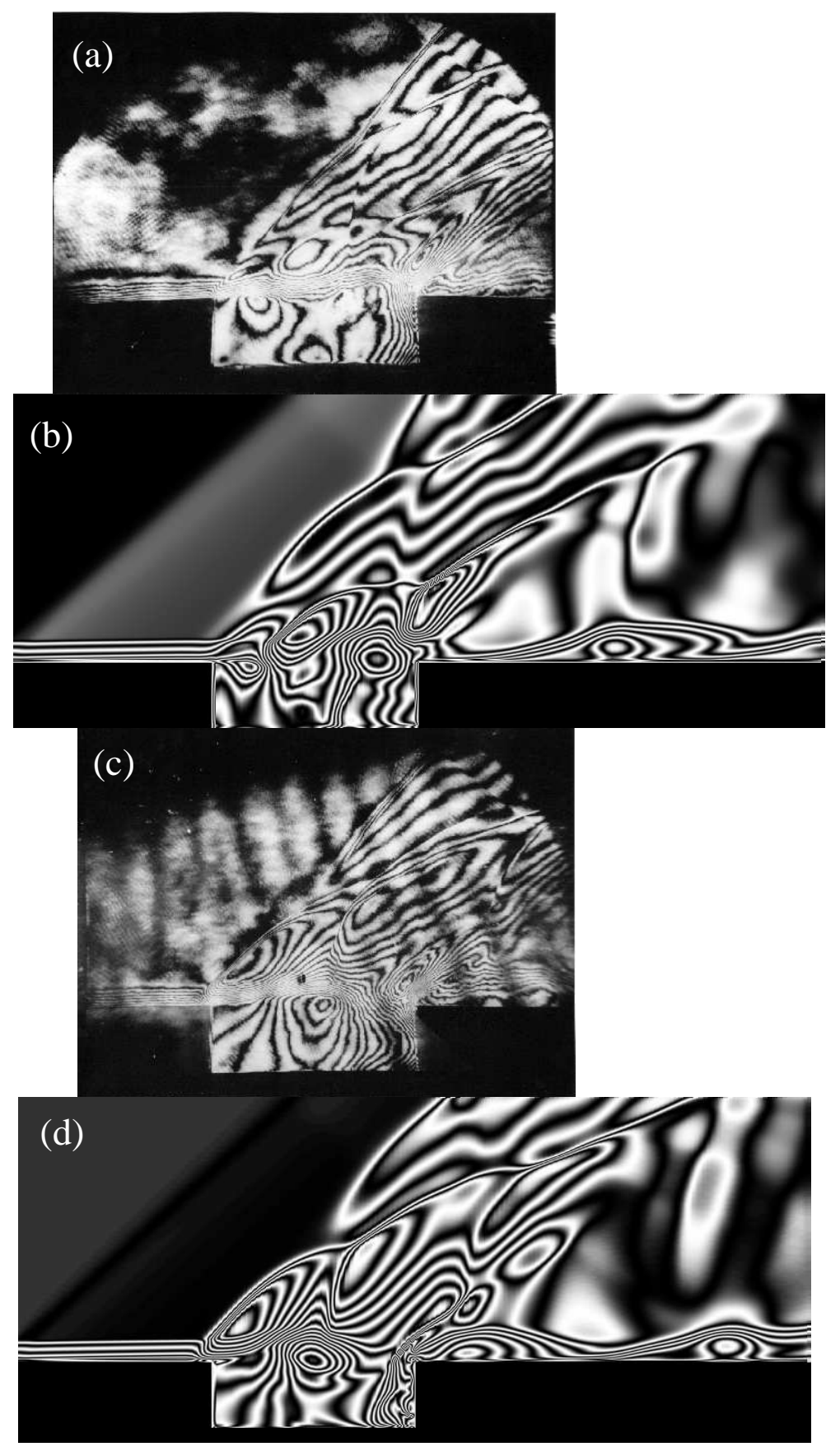

Fig. 9. $M_{\infty}=1.5$ cavity holographic interferograms. Mass ejection [34] (a) and prediction (b), mass entrainment [23] (c) and prediction (d). $\Delta \tilde{\rho}=0.03$. 


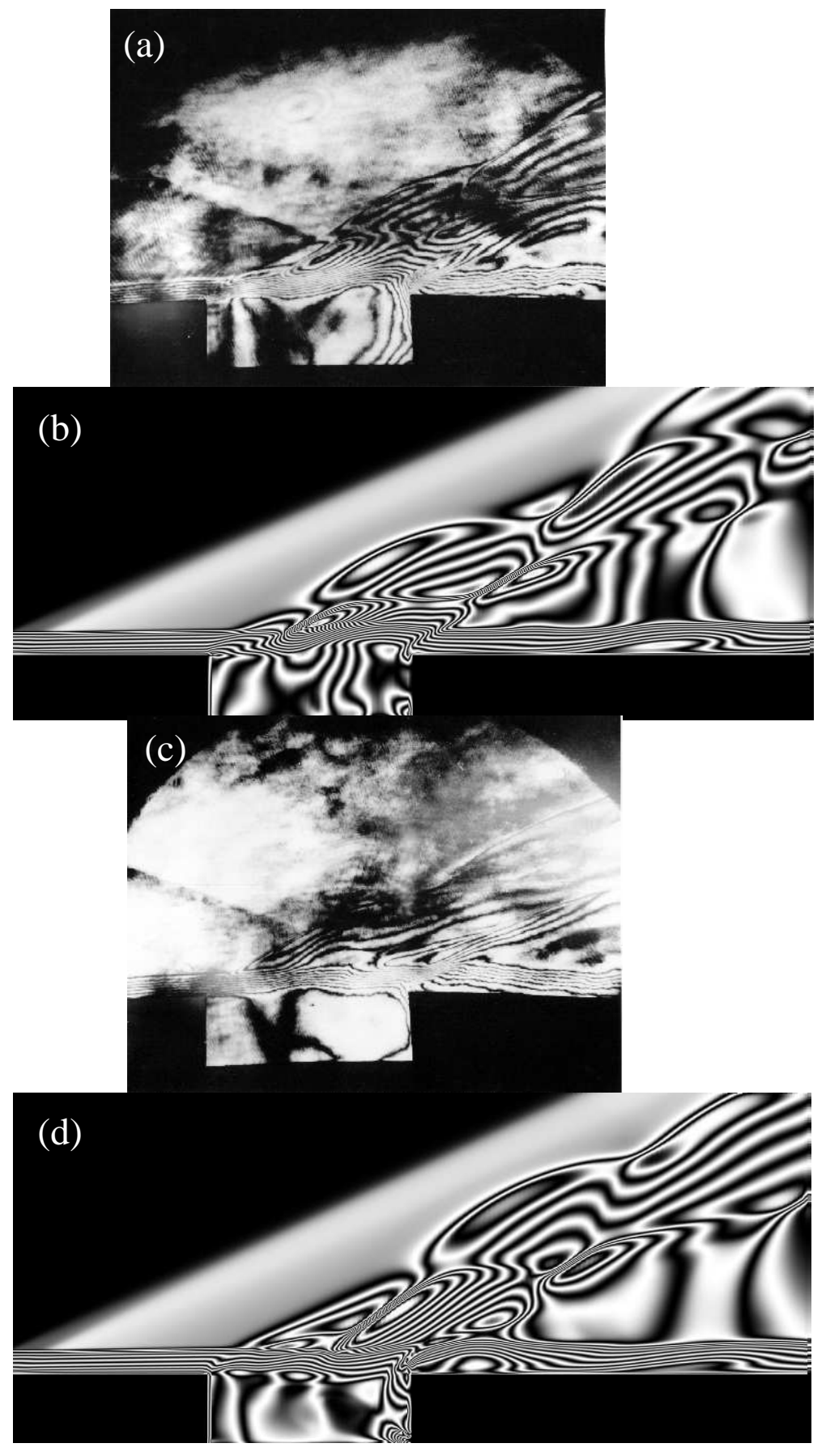

Fig. 10. $M_{\infty}=2.5$ cavity holographic interferograms. Mass ejection [34] (a) and prediction (b), mass entrainment [34] (c) and prediction (d). $\Delta \tilde{\rho}=0.03$. 

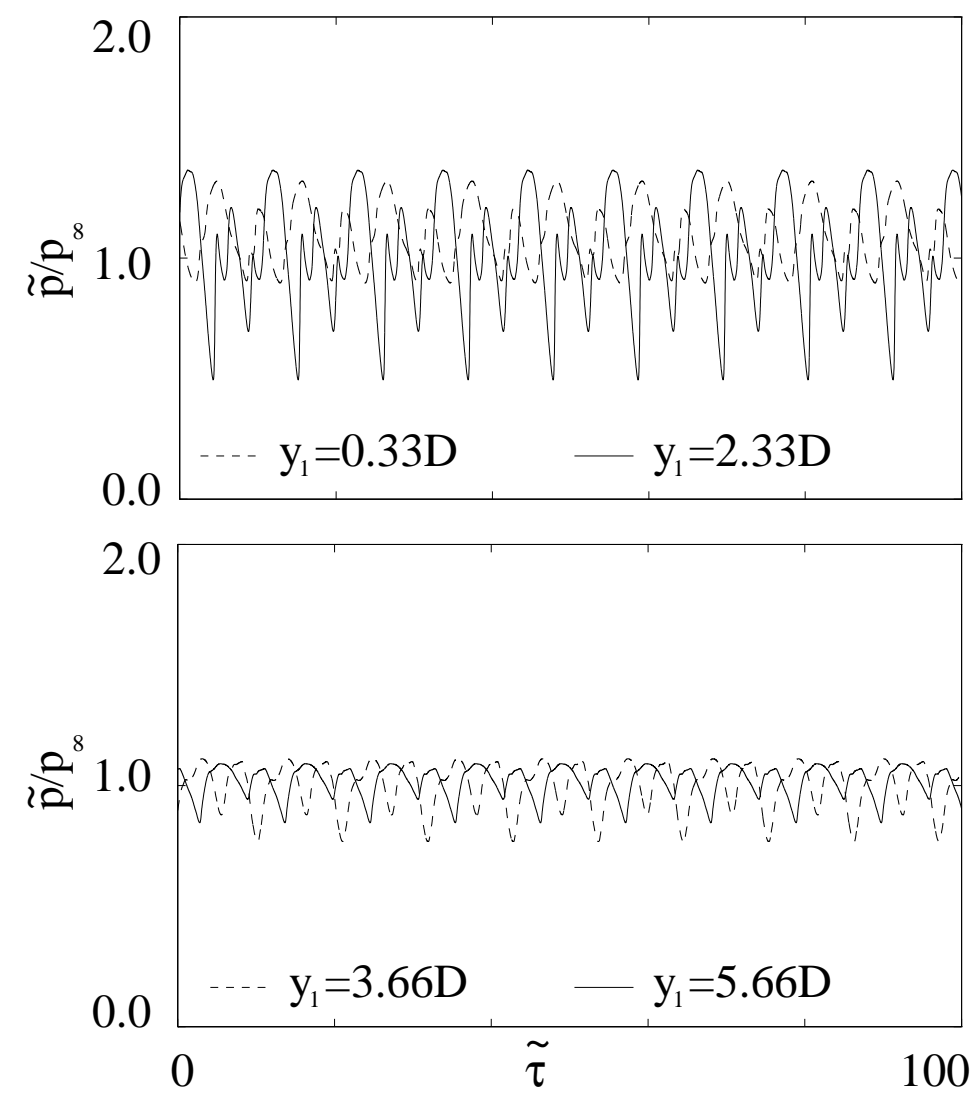

Fig. 11. $M_{\infty}=1.5$ short-time averaged pressure history. 

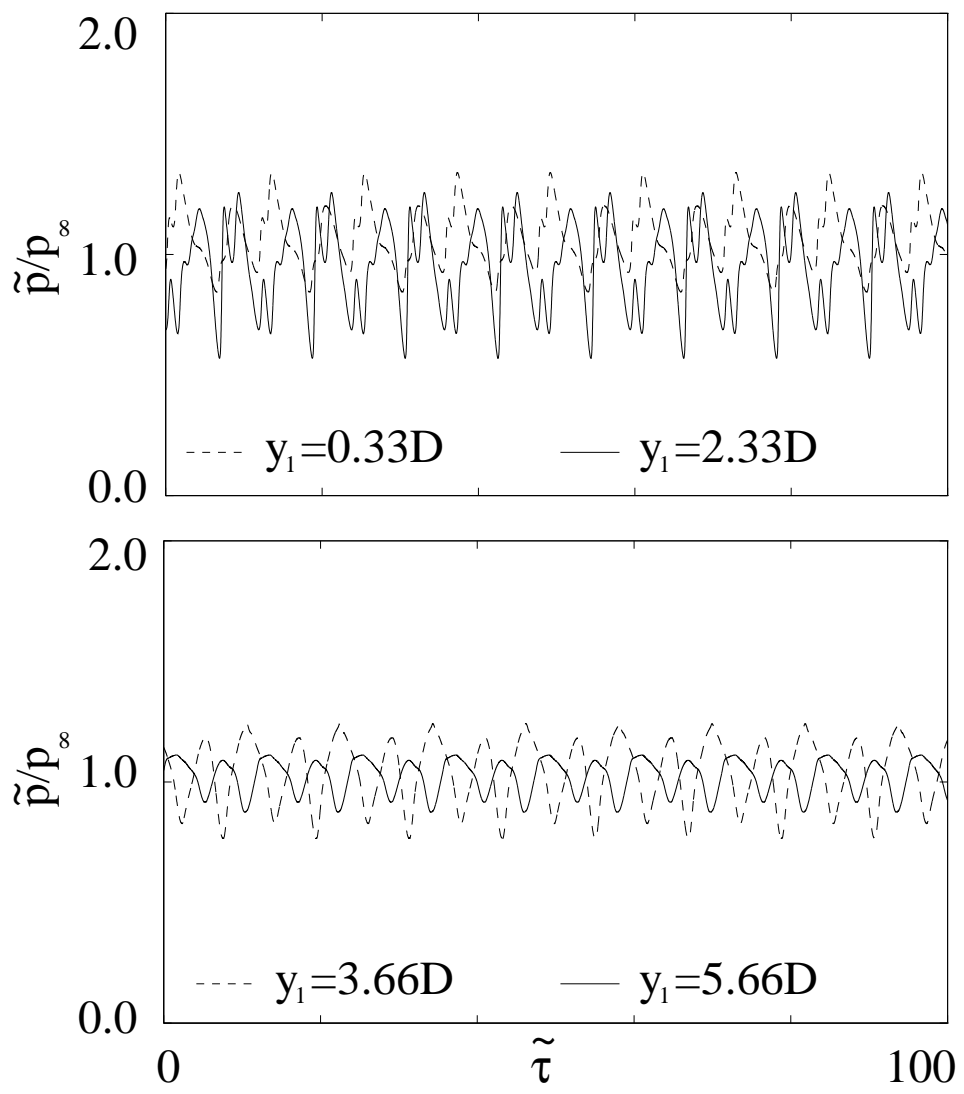

Fig. 12. $M_{\infty}=2.5$ short-time averaged pressure history.

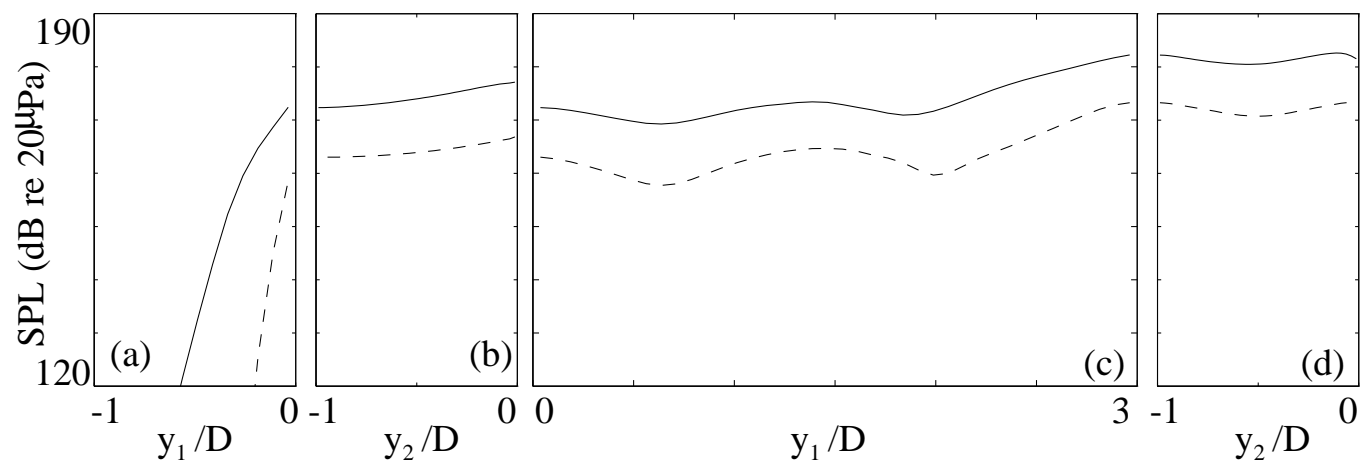

Fig. 13. Sound pressure level on the cavity walls. (-) $M_{\infty}=1.5,(--) M_{\infty}=2.5$. 


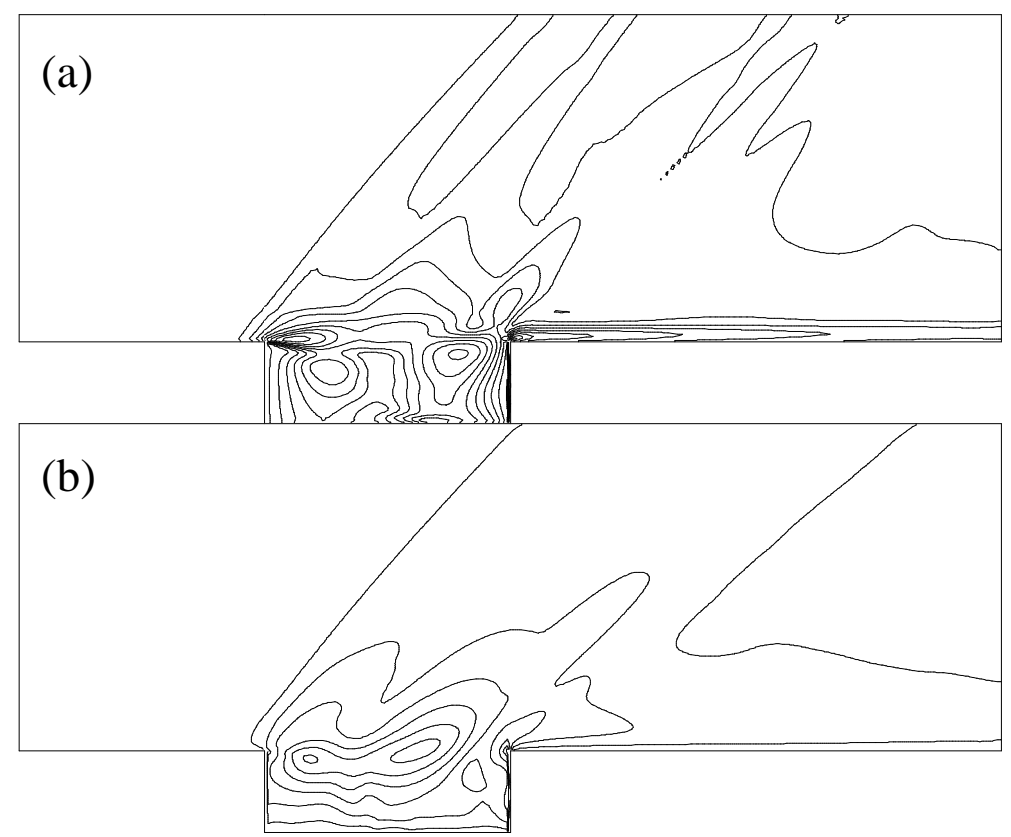

Fig. 14. $M_{\infty}=1.5$ cavity short-time root mean square velocity contours. (a) streamwise component, $\left[\left(\tilde{u}_{1}\right)_{r m s}\right]_{\max }=0.272$, (b) normal component, $\left[\left(\tilde{u}_{2}\right)_{r m s}\right]_{\max }=0.175 . \Delta \tilde{u}_{r m s}=0.03$.

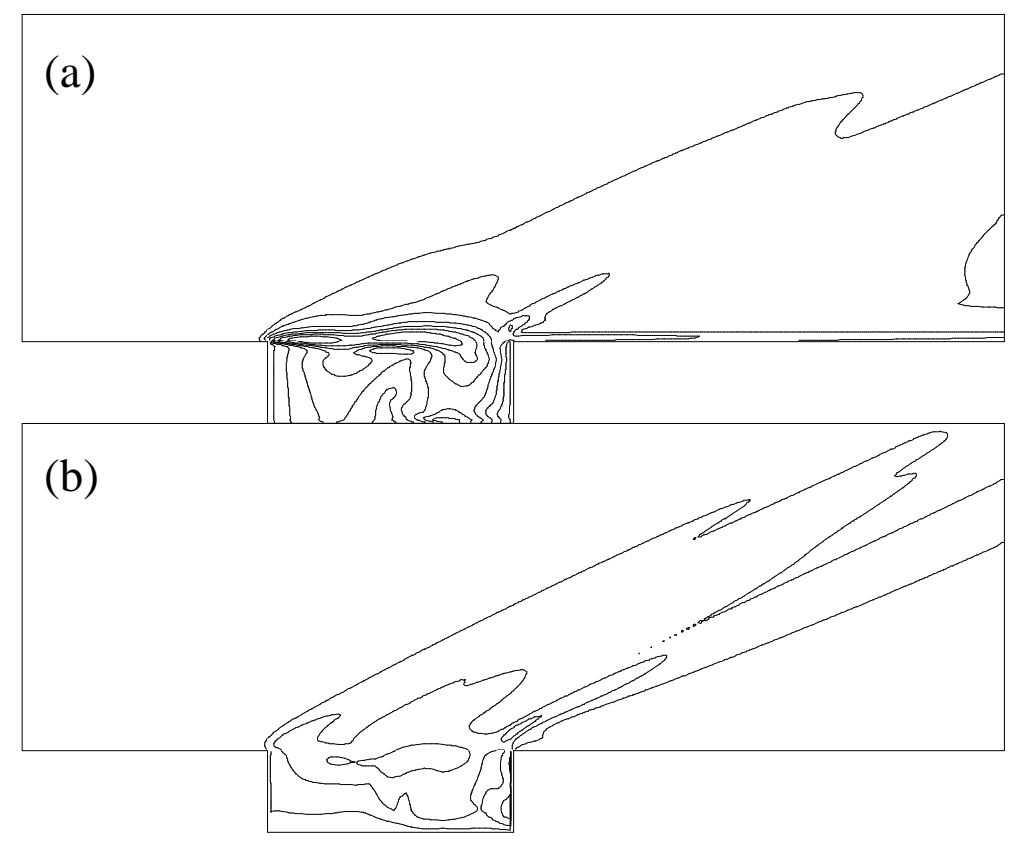

Fig. 15. $M_{\infty}=2.5$ cavity short-time root mean square velocity contours. (a) streamwise component, $\left[\left(\tilde{u}_{1}\right)_{r m s}\right]_{\max }=0.223$, (b) normal component, $\left[\left(\tilde{u}_{2}\right)_{r m s}\right]_{\max }=0.094 . \Delta \tilde{u}_{r m s}=0.03$. 

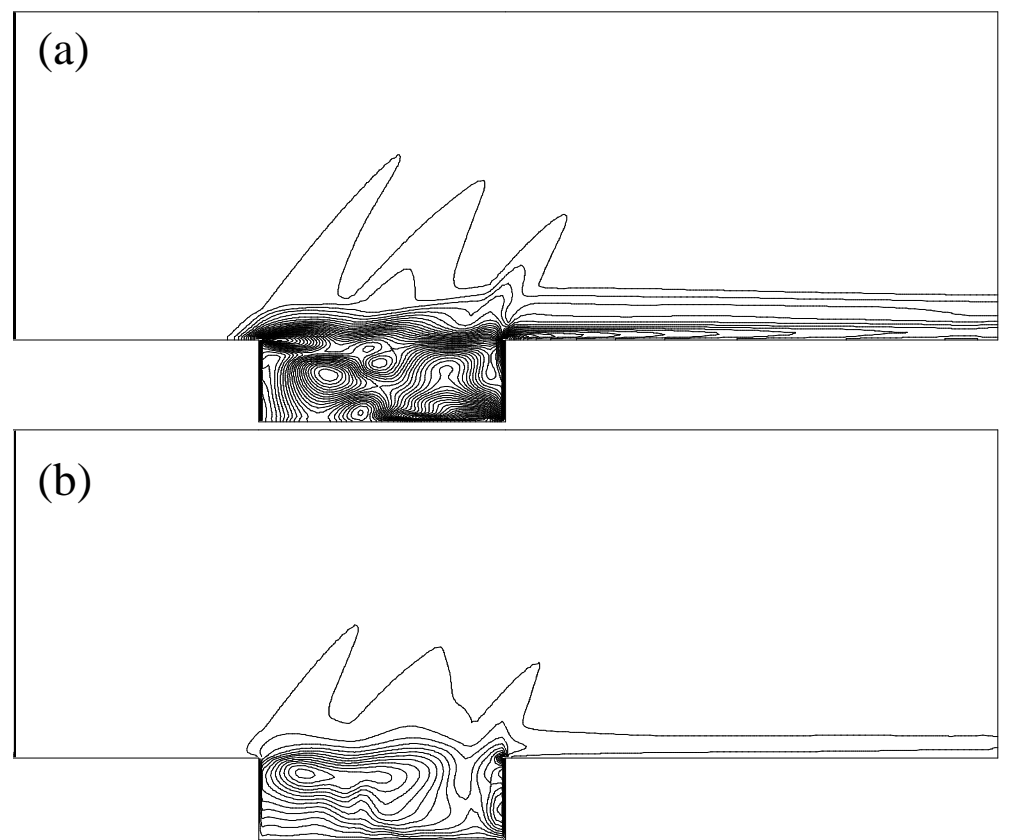

(c)

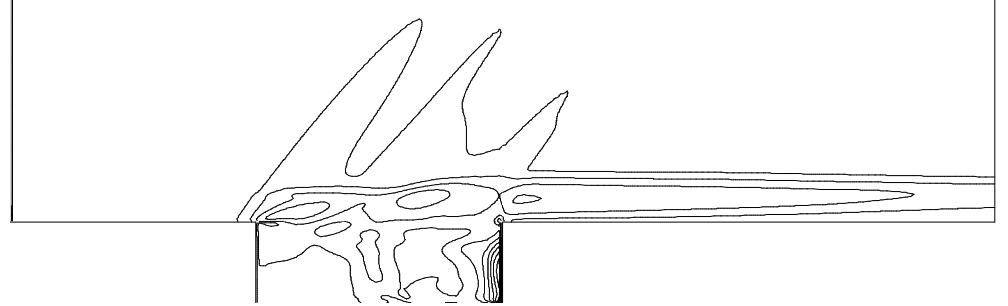

Fig. 16. $M_{\infty}=1.5$ cavity root mean square $\tilde{\boldsymbol{T}}$ contours. (a) $\left(\tilde{T}_{11}\right)_{r m s}$, $\left[\left(\tilde{T}_{11}\right)_{r m s}\right]_{\max }=0.480,(\mathrm{~b})\left(\tilde{T}_{12}\right)_{r m s},\left[\left(\tilde{T}_{12}\right)_{r m s}\right]_{\max }=0.130,(\mathrm{c})\left(\tilde{T}_{22}\right)_{r m s}$, $\left[\left(\tilde{T}_{22}\right)_{r m s}\right]_{\max }=0.083 . \Delta \tilde{\boldsymbol{T}}_{r m s}=0.01$. 

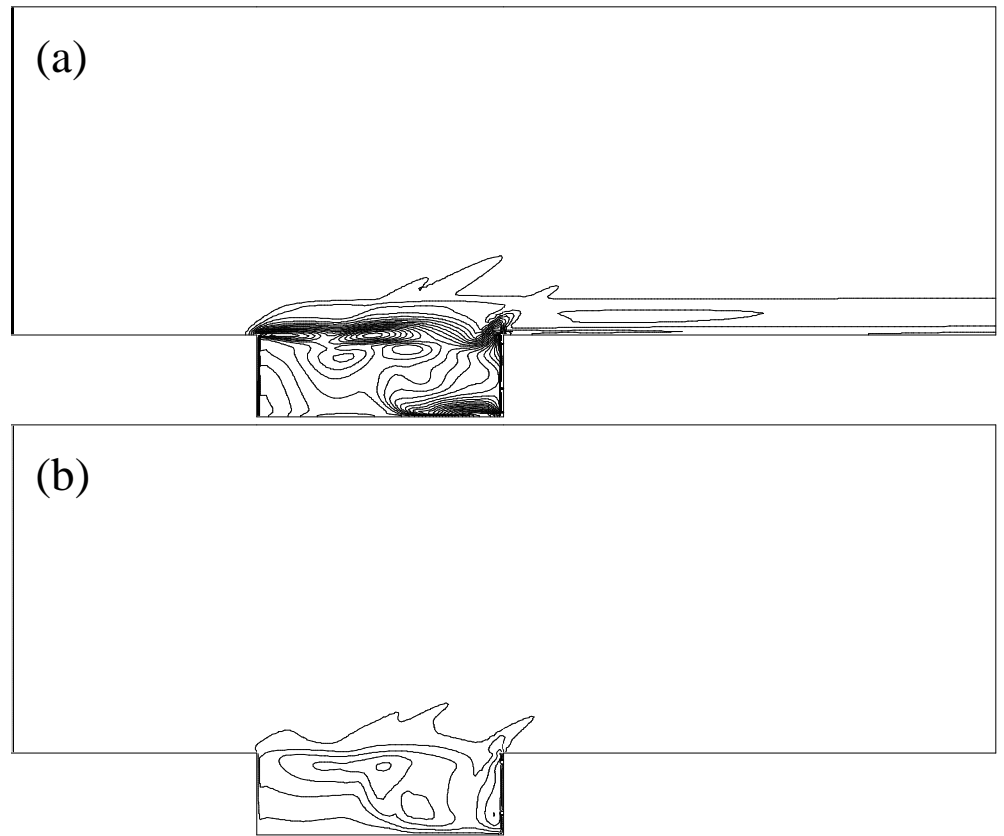

(c)

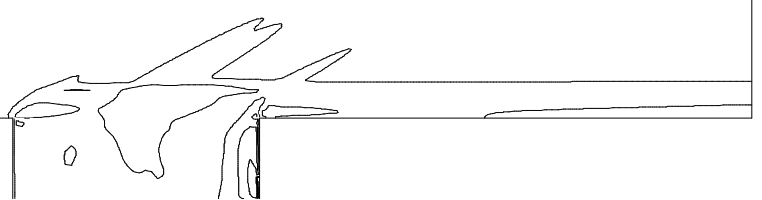

Fig. 17. $M_{\infty}=2.5$ cavity root mean square $\tilde{\boldsymbol{T}}$ contours. (a) $\left(\tilde{T}_{11}\right)_{r m s}$, $\left[\left(\tilde{T}_{11}\right)_{r m s}\right]_{\max }=0.304,(\mathrm{~b})\left(\tilde{T}_{12}\right)_{r m s},\left[\left(\tilde{T}_{12}\right)_{r m s}\right]_{\max }=0.046,(\mathrm{c})\left(\tilde{T}_{22}\right)_{r m s}$, $\left[\left(\tilde{T}_{22}\right)_{r m s}\right]_{\max }=0.042 . \Delta \tilde{\boldsymbol{T}}_{r m s}=0.01$. 